PUBLISHER'S NOTE: Based on the information available to BioMed Central, this article was apparently handled by Waseem Jerjes, an author of the article and Editor-in-Chief of the journal at that time. The manuscript was reviewed by two recent co-authors of Waseem Jerjes and accepted without revision.

\title{
RESEARCH
}

Open Access

\section{Spinal metastasis in thyroid cancer}

\author{
Sami Ramadan', Mohamed A Ugas², Richard J Berwick', Manisha Notay' ${ }^{1}$ Hyongyu Cho², \\ Waseem Jerjes $3,4,5,6^{*}$ and Peter V Giannoudis ${ }^{5,6^{*}}$
}

\begin{abstract}
Background: Thyroid carcinoma generally responds well to treatment and spinal metastasis is an uncommon feature. Many studies have looked at the management of spinal metastasis and proposed treatments, plans and algorithms. These range from well-established methods to potentially novel alternatives including bisphosphonates and vascular endothelial growth factor (VEGF) therapy, amongst others.

The purposes of this systematic review of the literature are twofold. Firstly we sought to analyse the proposed management options in the literature. Then, secondly, we endeavoured to make recommendations that might improve the prognosis of patients with spinal metastasis from thyroid carcinomas.
\end{abstract}

Methods: We conducted an extensive electronic literature review regarding the management of spinal metastasis of thyroid cancer.

Results: We found that there is a tangible lack of studies specifically analysing the management of spinal metastasis in thyroid cancer. Our results show that there are palliative and curative options in the management of spinal metastasis, in the forms of radioiodine ablation, surgery, selective embolisation, bisphosphonates and more recently the VEGF receptor targets.

Conclusions: The management of spinal metastasis from thyroid cancer should be multi-disciplinary. There is an absence; it seems, of a definitive protocol for treatment. Research shows increased survival with ${ }^{131}$ I avidity and complete bone metastasis resection. Early detection and treatment therefore are crucial. Studies suggest in those patients below the age of 45 years that treatment should be aggressive, and aim for cure. In those patients in whom curative treatment is not an option, palliative treatments are available.

\section{Introduction}

Thyroid cancer is uncommon, accounting for roughly $1 \%$ of all new malignant disease, about $0.5 \%$ of cancers in men and $1.5 \%$ in women [1-4]. It affects approximately 1900 people in the UK each year, but its incidence has been increasing for decades [5-8]. Much of the rise in incidence can be explained by improved diagnostic methods. However, this cannot entirely explain the upward trend [8-10].

Differentiated thyroid cancer (DTC) account for the vast majority $(90 \%)$ of all thyroid cancers and includes papillary $(70-75 \%)$ and follicular $(15-20 \%)$ cancers. Hürthle-cell cancers $(2 \%)$ and insular carcinomas also arise from follicular cells but are probably within the spectrum from differentiated to undifferentiated.

\footnotetext{
* Correspondence: waseem_wk1@yahoo.co.uk; pgiannoudi@aol.com ${ }^{3}$ Department of Surgery, Al-Yarmouk University College, Baghdad, Iraq ${ }^{5}$ Leeds Institute of Molecular Medicine, University of Leeds, London, UK Full list of author information is available at the end of the article
}

Undifferentiated carcinomas, which are anaplastic malignancies, account for $<5 \%$ of thyroid cancers. They derive from follicular cells. Medullary carcinoma, in contrast, constitutes $5-10 \%$ of thyroid cancers $[5,11,12]$.

The significance of the histological distinction relates to the management and prognosis of the different types of carcinomas. Differentiated carcinomas have a better prognosis than undifferentiated, Hürthle and insular cancers $[8,11]$. Medullary thyroid carcinomas arise from the $\mathrm{C}$-cells and are, therefore, aetiologically and clinically, different to follicular cancers $[8,11]$. There are also other rare types of thyroid malignancies including lymphomas and sarcomas.

In general, thyroid cancer is more prevalent in the middle-aged or old and in females [4]. Papillary malignancy typically occurs in young females and sometimes in children, whilst Follicular malignancy predominantly affects middle-aged females. Anaplastic thyroid cancer is more common in the elderly [7]. 
Papillary carcinoma is characterised by indolence and localised spread. It is known, however, to metastasize to both lung and bone. The prognosis of papillary thyroid cancer is good, especially in the young and if diagnosed early. Follicular malignancy preferentially metastasises to lung and bone. The prognosis is good if diagnosed early. Anaplastic thyroid carcinomas are very aggressive and locally invasive. They respond poorly to treatment and prognosis is poor. Medullary cancer sometimes has a familial origin associated with the RET gene and can also be part of Multiple Endocrine Neoplasia type 2 syndrome (MEN-2 syndrome). It is too, characterised by indolence and localised spread, as well as metastasis to different sites. It has a poor prognosis but can be treated if found before distant spread $[3,5,12,13]$.

In $90 \%$ of cases, thyroid carcinoma presents simply as thyroid nodules. Rarely, the first manifestation is cervical lymphadenopathy (5\%), or distant metastasis in the lungs, bone, liver or brain. Bony metastasis occurs in approximately $2-13 \%$ of people with thyroid malignancy; the proportion is overwhelmingly follicular, and many of those develop in the spine $[3,13,14]$. The latter are particularly debilitating as they often cause pain, fractures, spinal cord compressions and affect mobility and quality of life (QoL) [15]. The remission rate in bony metastasis is $7-20 \%$ [16].

The survival rate in thyroid carcinoma is generally good, apart for the anaplastic type. The 10-year survival rate in DTC is $80-95 \%$. However, this figure drops to about $40 \%$ when distant metastasis is present [16].

The pathogenesis of thyroid carcinomas is not fully understood. Aetiology is known only in a few rare cases of familial papillary and medullary carcinomas, and in some patients who have been exposed to irradiation or radioactivity [5].

There is a pathway to diagnose thyroid cancer including: physical exam to feel for nodules, blood tests (particularly: TSH, thyroglobulin and calcitonin levels), ultrasound, thyroid scan with a radioactive marker. Also, biopsy with fine needle aspiration (FNA) is helpful; however, surgery is the gold standard and the only conclusive diagnostic method $[4,10,17]$.

\section{Management of thyroid cancer}

It is widely agreed in the literature that surgical resection should be the initial step in treating most thyroid cancers [4,17-19]. Following a biopsy to determine the histology of the tumour, a decision is made whether to perform a lobectomy or total thyroidectomy $[4,19]$. Patients who have had total or near-total thyroidectomy are required to have their serum thyroglobulin checked no sooner than six weeks after the procedure in order to check for recurrence.
The surgery is often followed by adjuvant radioiodine therapy $\left({ }^{131} \mathrm{I}\right.$ ablation). Following the resection of the thyroid gland, thyroid hormone replacement therapy is given in order to rectify the resulting hypothyroidism. It is also thought that in high doses the replacement therapy helps prevent recurrence of the cancer [19].

Chemotherapy and external radiation are also used in the treatment albeit more rarely than the above mentioned treatments. External radiation is used infrequently as palliative treatment in cases of unresectable or secondary disease $[10,11,17,19]$.

The use of chemotherapy is more controversial. It is generally ineffective in most types of thyroid cancers, but it is indicated for the anaplastic type, lymphoma or metastatic tumour. It has also been used as palliation in end-stage disease unresponsive to irradiation and also for progressive, symptomatic disseminated cancer [20]. The agents used are doxorubicin and cisplatinum $[11,17,19]$.

In most cases the treatment of thyroid carcinomas results in long-term remission. Survival is excellent in younger patients with recurrence more likely at extremes of age $[21,22]$. However, there are still patients with poor outcomes. They are usually the patients with metastasis and/or advanced disease at diagnosis $[11,18]$. For this reason, new treatment modalities are being explored.

\section{Metastasis from thyroid cancer}

DTC metastasises preferentially to bone (25\%) second only to the lung (49\%) whilst synchronous spread to lung and bone accounts for a further $15 \%$ of distant metastasis of DTC. The remaining occur in other soft tissues $[3,23,24]$.

Bone metastasis occurs in $2-13 \%$ of DTC cases. They are more prevalent in follicular cancer $(7-28 \%)$ than in papillary cancer (1.4-7\%) [3]. The great majority of bone metastasis occur in regions where blood flow is high, such as the axial skeleton red marrow in vertebrae, ribs and hips [3]. The spinal lesions are mostly osteolytic, with new bone forming in response to the bone destruction [3].

The diagnostic modalities used in spinal metastasis are different to those used in the determination of thyroid cancer. CT and MRI scans are used to provide high resolution imaging of the spine. MRI is better at differentiating soft tissue structures and, therefore, it is slightly more sensitive at detecting bone marrow metastasis ${ }^{26}$. Bone scintigraphy and other nuclear studies, such as FDG-PET and SPECT, are also used for localisation of lesions and have high sensitivity and specificity [25].

When bony metastasis is present, the 10-year survival rate is estimated between $13 \%$ and $21 \%$, which is roughly equivalent to a $50 \%$ reduction compared to nonmetastasised cancer ${ }^{3}$. It is often the case that the lesion (s) do not respond to curative treatment and palliative 
treatment at diagnosis remains the only option; the aim being to improve the quality of life [3].

\section{Spinal metastasis}

Bone, after the lungs and liver is the third commonest site of metastasis [26]. Spinal metastasis is by far the most common type of spine tumours, outnumbering primary spinal neoplasm by more than twenty-to-one [25], and, are overwhelmingly breast, lung, prostate or renal primaries in origin [25-27]. This owes much to their respective incidence as well as their special predilection for the vertebral column [25].

Prevalence of spinal metastasis (SM) is highest among individuals between the $4^{\text {th }}$ and $7^{\text {th }}$ decade of life $[25,27,28]$. Males are more likely to be afflicted; this is thought to be reflective of the higher prevalence of lung cancer in this group [12] and the higher prevalence of prostate cancer relative to breast cancer in females [25].

At the time of initial diagnosis, $1 \%-3 \%$ of patients with thyroid cancer have distant metastasis whereas another 7\%-23\% will develop distant metastasis during the course of their disease $(2,5-8,10-12)$. Bone metastasis is diagnosed clinically in $2 \%-13 \%$ of patients with differentiated thyroid cancer [29].

Well-differentiated thyroid cancers (DTC) account for the vast majority (85-98\%) of thyroid malignancies. Bone metastasis incidence in differentiated thyroid cancers (DTC) is $2-13 \%$ [30,31]. Papillary thyroid cancers (PTC) accounts for $77 \%$ of DTC and has a low incidence of SM of 1-7\% [31] whilst follicular thyroid cancer (FTC) which accounts for $15 \%$ of all DTC has an incidence of bone metastasis of 7-20\% [31]. Hürthle cell carcinomas, which accounts account for $2 \%$ of thyroid malignancies, have the highest propensity $(12 \%)$ to metastasize to spine.

Spinal metastasis typically affect the thoracic (60-80\%), lumbar $(15-30 \%)$ and cervical spine $(<10 \%)$ [32-54] with the preferred route of metastasis to the spine being via the arterial or venous -Batson's venous plexus - vessels resulting in multifocal lesions. Direct infiltration from paraspinous disease or, less commonly, through the cerebrospinal fluid $[25,32]$ are also potential routes of metastasis. The vertebral body (85\%) [54] is the commonest site for initial involvement; the posterior aspect of which is preferentially involved (66\%) [25]. The paravertebral spaces (10-15\%) and the epidural space $(<5 \%)$ are also initial sites of metastatic involvement $[25,32,54]$.

Vertebral metastasis are asymptomatic and may be incidental findings following routine bone scans in patients presenting with systemic disease $[32,33]$. Classical clinical symptoms develop with the progression of spinal metastatic disease and are consequences of metastatic infiltration and/or compression of paravertebral, osseous and neural tissue [33].
Spinal canal to spinal cord ratio is smallest in the thoracic spine hence spinal cord compression is more common in the thoracic spine [33]. The most frequent cause of spinal cord compression and nerve root compression is the expulsion of metastatic tissue and/or detritus of bone into the spinal canal or neural foramina following metastatic infiltration and ensuing partial collapse of the vertebral body. On infrequent occasion, metastatic tissue may break into the spinal canal and cause spinal cord compression without assaulting the vertebral body's structural integrity.

The chief presenting symptom of spinal metastasis is pain $(83-95 \%)$ [25,32]. Patients often present with localised, gradual onset pain caused by periosteal stretching and inflammation [32]. It is invariably progressive and unremitting in nature, often worse at night. It may improve with activity and anti-inflammatory medications. The affected area is typically tender on examination [33].

Radicular pain, described as shooting pain, is a common compliant in SM. It is caused by impingement or irritation of nerve roots in the intervertebral foramina, either by direct tumour compression or via tumour induced pathological fractures. Neuropathic pain characterised by an intense, burning sensation is more common in intradural metastasis [32].

Metastatic invasion of the spinal canal occurs predominantly in an anterior direction. Spreading via the posterior aspect of the vertebral body [33], deficiencies in motor functions comprise the second most common presenting symptom $\left(35-75 \%{ }^{26}\right)$ in patients with vertebral column metastasis $[32,33]$. Limb weakness is a common symptom, which is seen also on physical examination [25].

Posterior displacement of the spinal cord and impingement against the lamina may result in sensory dysfunction $[32,33]$. It is a feature of advanced spinal metastatic disease and can be accompanied by profound motor dysfunctions such as anal and urethral sphincter dysfunction and sexual malfunction [25,32].

These "red flag" signs and symptoms should prompt SM diagnosis in thyroid cancer patients. In such patients, a full diagnostic workup should be instigated to ascertain the level(s) of vertebral column involvement, spinal instability and the degree of neurological impairment. These parameters influence the decision to operate or not $[25,32,33]$.

Plain X-ray is used to identify metastatic lesion(s), tumour mass(s) and evaluation of spinal stability [27,32]. Spinal instability is indicated by progressive deformity, significant angulation or translocation and/or $>50 \%$ involvement of the vertebral body. Cervical disk space narrowing is an important maker of vertebral collapse. Metastatic lesions are indicated by vertebral body involvement, 
erosion of the spinous process and the inability to fully visualize pedicles [27]. X-rays are insensitive in early spinal metastatic diagnosis, however, because a $30-50 \%$ demineralisation of bone is required before lytic lesions become apparent on radiographic film $[25,27,32]$.

Magnetic resonance imaging (MRI) is the gold-standard imaging modality in SM diagnosis. It renders exquisitely detailed multiplanar imaging, allowing the visualisation of metastatic infiltration and/or compression of paravertebral, osseous and neural tissue [25,27,32]. T1- and T2-weighted imaging as well as contrast-enhanced and fat-suppressed studies in all three planes aid diagnosis ${ }^{33}$.

Computed tomography (CT) imaging is an excellent modality in assessing the osseous spine. It has a high degree of accuracy ( $90 \%$ sensitivity, 100\% specificity) [28] in identifying metastatic lesions, vertebral destruction and spinal stability. CT angiography is ideal in identifying spinal metastasis from highly vascular primary malignancies such as thyroid cancers [32].

Bone scintigraphy is used to screen for bone metastasis. Despite its documented 62-89\% [25] sensitivity, it should be noted that bone scintigraphy measures abnormalities in bone metabolism and does not, therefore, possess a high specificity in identifying SMs.

MRI and/or CT should be used to authenticate suspected SM. Single-photon emission computed tomography (SPECT) and fluorodeoxyglucose positron emission tomography (FDG-PET) [25] are both superior to bone scintigraphy and are used in surveillance of patients suspected of SM. Finally biopsy under CT fluoroscopic guidance is crucial in staging SM and formulating surgical treatment plan.

The purposes of this systematic review of the literature are twofold. Firstly we sought to analyse the proposed management options in the literature. Then, secondly, we endeavoured to make recommendations that might improve the prognosis of patients with spinal metastasis from thyroid carcinomas.

\section{Materials and methods}

A thorough electronic literature search was conducted using online journal databases. These included PubMed, Google Scholar, Web of Knowledge, Science Direct and

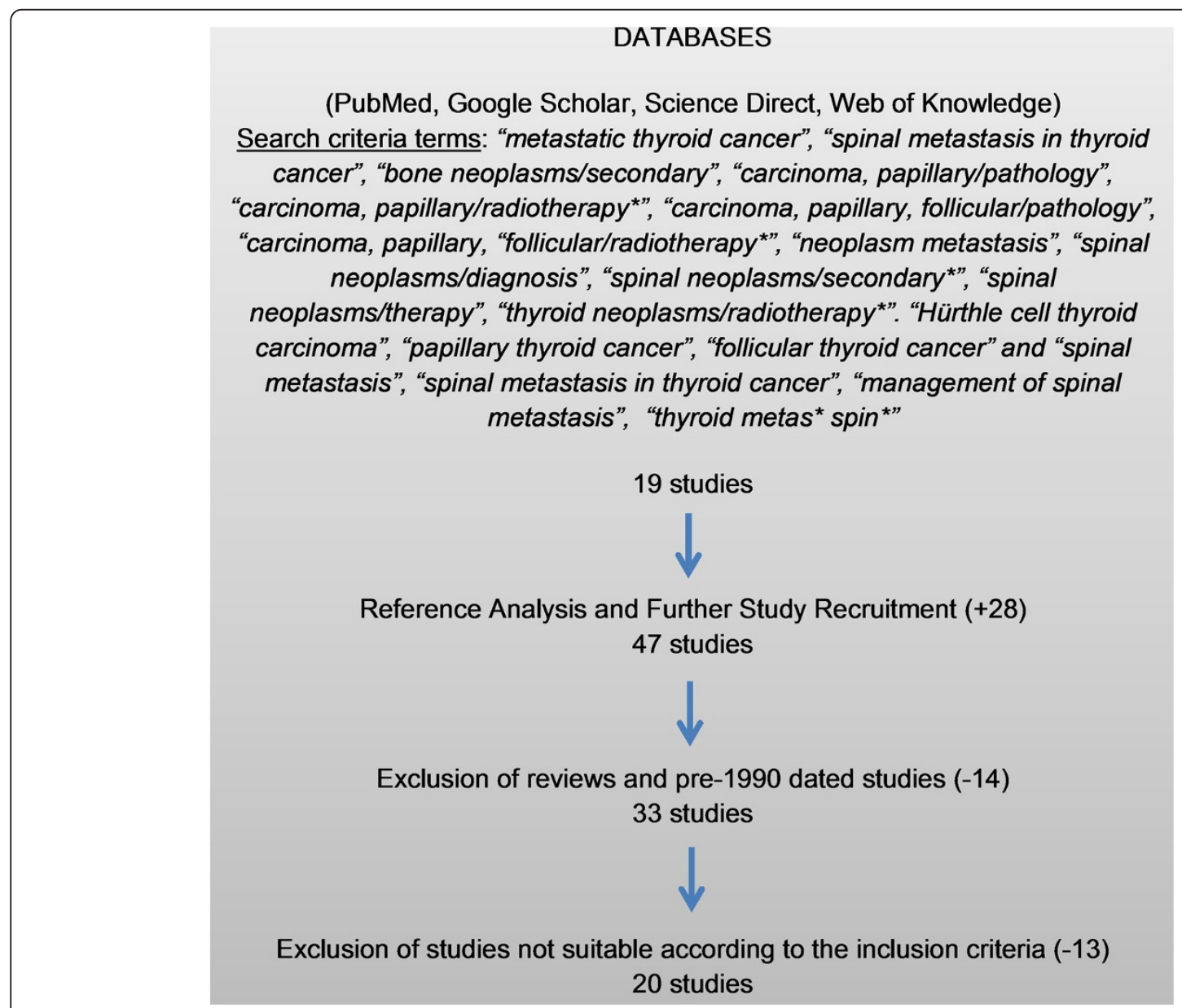

Figure 1 Flow chart illustrating search protocol. 
MEDLINE. Our search criteria terms were broad including, "spinal metastasis in thyroid cancer", "management of spinal metastasis", "thyroid metas* spin"”. This yielded 20 studies. To supplement our research, we reviewed the references of the literature initially found, in order to avoid neglecting relevant articles that were missed by our search criteria. We excluded from our initial pool of recruited studies those that were review papers and those articles dated pre 1990 to ensure a body of contemporary literature.

Articles were deemed pertinent if they described metastatic thyroid cancer of a follicular, papillary or Hürthle nature. We sought, particularly, studies that included a population of patients with bone metastasis and spinal involvement. We did not include small case reports or studies that investigated spinal metastasis, without mention of thyroid cancer because the nature of thyroid cancer treatment is specific. In order to attain reliably representative results, we eliminated case reviews. The chosen studies were selected by two independent reviewers using the above protocol.

\section{Results}

Our search protocol refined our pool of articles to 20 [13-16,29,30,34-47], as seen in Figure 1. In this systematic review we focused on therapeutic management of spinal metastasis in thyroid cancer with reference to survival time, pain management and prognostic indicators. As a part of this review, the various parameters were evaluated including the study design, sample size, histological type of cancer, mean age, outcome and mortality. The characteristics of the studies can be seen in Table 1.

Our search protocol retrieved 20 studies published from 1996 to 2012 (Table 1). Studies investigated the use of conventional and novel intervention for distant and spinal metastasis, including radioactive iodine, surgery, selective embolisation therapy (SET), small molecule inhibitors and bisphosphonates. Reported outcomes included survival rates, morbidity and prognostic factors associated with each intervention. There were 16 retrospective cohort studies $[13,15,16,29,30,34,36,38-45,47], 1$ observational study [14], 1 prospective interventional study [35], 1 Phase II trial [37] and 1 Phase III clinical trial [17].

Reported cases of spinal metastasis and therapeutic modalities used can be seen in Table 2. Summary results pertaining to the use of conventional and novel therapeutic modalities in treating patients with distant metastasis from thyroid carcinoma can be seen in Tables 3 and 4, respectively. The population size in each study varied considerably between studies, from Durante et al. [30] $(\mathrm{n}=444)$ to Van Tol et al. [44] $(\mathrm{n}=11)$.

One of our studies, by Wells et al. [46], was a randomized controlled trial, which investigated the effect of vandetanib versus placebo, however, there were no interventional trials comparing the effect of different treatments of metastatic disease. Of the retrospective trials, too, no studies sought to compare treatments.

Two studies looking at the specific use of bisphosphonates were Orita et al.[15] and Vitale et al. [45], although Orita et al. had mentioned, previously, that bisphosphonates contribute to an improved 5-year survival (52\% with and $30 \%$ without bisphosphonate; $P$ value $=0.11$ ). However, on subsequent interrogation it was found that zolendronic acid infusion once a month, increased the chance of avoiding skeletal-related events (SREs), defined as bone fracture, spinal cord compression, and hypercalcaemia, in the first 3 years post detection $(50 \%$ without bisphosphonate compared to $86 \%$ with bisphosphonate; $P$ value of 0.002 ). This corroborated Vitale et al.'s work that illustrated pamidronate IV infusion once a month, for 12 months, improves the quality of life using the FACT-G scoring system $(P$ value $=0.0059)$ [45].

Surgical intervention for bone metastasis was examined in 4 studies: Bernier et al. [34], Demura et al. [13], Orita et al. [38] and Quan et al. [40]. Bernier et al., showed through multivariate analyses that complete SM surgery was an independent prognostic indicator of improved survival, however, this only applied to younger patients (under 45 years of age) [34]. Demura et al., who employed an en-bloc resection approach, reported that the survival rate from surgery was $74 \%$ at 5 years and $25 \%$ at 10 years. The risk of local recurrence after debulking surgery was $57 \%$ and required further surgery at an average of 41 months, however recurrence after total en bloc spondylectomy (TES) was $10 \%$ and this difference was statistically significant. They reported that all patients alive at study close were neurologically preserved [13]. Orita et al. reported 5-year survival with surgery of $60 \%$ compared to $37 \%(P$ value $=0.05)$ with no surgery [38].

Selective Embolisation therapy (SET) therapy was investigated in 3 studies, by Van Tol et al. [44], Eustatia Rutten et al. [16] and, most recently, by De Vries et al. [36]. Van Tol et al. [15] found that those who received combined embolisation with 131-I therapy, serum $\mathrm{Tg}$ fell by $88.7 \%$, which was significantly more than the control group 16.6\%; $\mathrm{p}<0.05$. Eustatia-Rutten et al. found it was effective at palliation in $59 \%$ of cases, with success duration being 6.5 months alone, but for embolisations combined with additional radioiodine or external irradiation, this was increased to 15 months $(\mathrm{P}>0.0146)$ [16]. Finally, De Vries et al., came to the conclusion that survival is not significantly improved, however symptoms were reduced in 55\% of procedures [36].

The impact of ${ }^{131}$ I therapy was investigated in 5 studies [14,29,38,39,41]. Although Pittas et al. [29] did not find an increased survival in those who were treated with ${ }^{131} \mathrm{I}$ for 
Table 1 Summary of the study characteristics

\begin{tabular}{|c|c|c|}
\hline Study, year & Study design & End point \\
\hline Bernier et al., $2001^{35}$ & Retrospective cohort analysis & Survival and impact of treatment on survival. \\
\hline Cabanillas et al., $2010^{36}$ & Prospective intervention & $\begin{array}{l}\text { Determine response and progression free survival due } \\
\text { to treatment with sorafenib/sunitinib }\end{array}$ \\
\hline De Vries et al., 2008 37 & Retrospective cohort analysis & $\begin{array}{l}\text { Determine the effect of selective embolisation on serum } \\
\text { thyroglobulin and symptoms }\end{array}$ \\
\hline Demura et al., $2011^{14}$ & Retrospective cohort analysis & $\begin{array}{l}\text { Determine the effect of total en bloc spondylectomy on } \\
\text { survival and local recurrence }\end{array}$ \\
\hline Durante et al., $2006^{31}$ & Retrospective cohort analysis & $\begin{array}{l}\text { Determine the response and effect on survival of } \\
{ }^{131} \text { | therapy }\end{array}$ \\
\hline Eustatia-Rutten et al., $2003^{17}$ & Retrospective cohort analysis & $\begin{array}{l}\text { Determine the effect of palliative embolisation on } \\
\text { clinical symptoms. }\end{array}$ \\
\hline Hindie í et al., $2007^{15}$ & Observational study & $\begin{array}{l}\text { To investigate the impact of early }{ }^{131} 1 \text {-based detection } \\
\text { on outcome. }\end{array}$ \\
\hline Kloos et al., $2009^{38}$ & Phase II Trial & Objective response rate of VEGFR inhibitors \\
\hline Orita et al., $2011^{16}$ & Retrospective cohort analysis & $\begin{array}{l}\text { Determine the efficacy of zolendronic acid on skeletal-related } \\
\text { events and bone metastasis from thyroid cancer }\end{array}$ \\
\hline Orita et al., $2010^{39}$ & Retrospective cohort analysis & Determine prognostic factors and analyse treatment methods \\
\hline Petrich et al., $2000^{40}$ & Retrospective cohort analysis & $\begin{array}{l}\text { Determine the therapeutic outcome and side effects of } \\
\text { radioiodine in bone metastasis from thyroid cancer }\end{array}$ \\
\hline Pittas et al., $2000^{30}$ & Retrospective cohort analysis & Determine prognostic indicators and impact of treatment on survival \\
\hline Quan et al., $2012^{41}$ & Retrospective cohort analysis & $\begin{array}{l}\text { Determine survival of patients with vertebral metastasis from } \\
\text { thyroid cancer }\end{array}$ \\
\hline Qiu et al., $2011^{42}$ & Retrospective cohort analysis & Determine the efficacy of ${ }^{131}$ I therapy and survival \\
\hline Schlumberger et al., $1996^{43}$ & Retrospective cohort analysis & Determine the response of bone and lung metastases to ${ }^{131}$ | \\
\hline Stojadinovic et al., $2002^{44}$ & Retrospective cohort analysis & $\begin{array}{l}\text { Determine distant disease free survival and post metastatic disease } \\
\text { specific survival after surgery for distantly metastatic thyroid carcinoma }\end{array}$ \\
\hline Van Tol et al., $2000^{45}$ & Retrospective cohort analysis & $\begin{array}{l}\text { Determine the effect of embolisation in combination with } \\
\text { radioiodine in terms of pain, serum T and neurological symptoms }\end{array}$ \\
\hline Vitale et al., $2001^{46}$ & Retrospective cohort analysis & $\begin{array}{l}\text { Determine the impact of pamidronate on bone pain, performance } \\
\text { status and evaluate the degree of tumour response. }\end{array}$ \\
\hline Wells et al., $2012^{47}$ & Phase III RCT & $\begin{array}{l}\text { Determine progression free survival of patients on vandetanib for } \\
\text { metastatic thyroid cancer }\end{array}$ \\
\hline Zettinig et al., $2002^{48}$ & Retrospective cohort analysis & Determine the role of surgery on survival of metastatic thyroid cancer \\
\hline
\end{tabular}

bony metastasis, it was suggested that it may be beneficial in patients whose bony metastases show ${ }^{131}$ I uptake. Petrich et al. [39] showed positive radioiodine uptake by metastases significantly increased survival compared to those individuals who had negative uptake. Orita et al. [38] and Hindie et al. [14] provided findings that a significant survival advantage was obtained with ${ }^{131} \mathrm{I}$ therapy supporting that claim. Additionally, a study by Qiu et al. [41] found that ${ }^{131}$ I therapy was $63.9 \%$ effective at reducing symptoms due to bony metastasis such as pain, and could therefore be used as a palliative agent in patients with multiple bone metastases.

VEGFR therapy was investigated in two studies $[37,46]$ in the form of sorafenib and vandetanib. Kloos et al. [37] found that sorafenib caused stabilisation of disease progression for greater than 6 months in $56 \%$ of patients
(95\% CI, 40 to 72). Wells et al. [46] investigated vandetanib in the form of a phase III randomised control trial and investigated the progression free survival (PFS) in comparison with placebo. They found a prolonged progression free survival in the treatment group estimated at 30.5 months, compared a PFS of 19.3 months in the placebo group. However, $12 \%$ of patients stopped the drug due to adverse events [46].

\section{Discussion}

Metastatic thyroid tumours cause considerable morbidity [48]. They are also indicative of advanced disease and are associated with a poor prognosis [29] and a reduction in treatment response. However, comparatively, they have a more favourable prognosis of most tumours, which metastasize to the spine $[40,49]$. Tumour invasion 
Table 2 Clinical characteristics at diagnosis of patients with spinal metastasis from thyroid carcinoma and the therapeutic strategies deployed

\begin{tabular}{|c|c|c|c|c|c|c|c|c|c|}
\hline $\begin{array}{l}\text { Author, } \\
\text { Year }\end{array}$ & $\begin{array}{l}\text { Patient } \\
\text { No. }\end{array}$ & $\begin{array}{l}\text { Age (yrs), sex } \\
\text { at time of } \\
\text { symptomatic } \\
\text { spine disease }\end{array}$ & $\begin{array}{l}\text { Thyroid } \\
\text { cancer } \\
\text { histology }\end{array}$ & $\begin{array}{l}\text { TNM } \\
\text { stage }\end{array}$ & $\begin{array}{l}\text { Intervention for } \\
\text { primary site }\end{array}$ & $\begin{array}{l}\text { Location } \\
\text { of spinal } \\
\text { metastasis }\end{array}$ & $\begin{array}{l}\text { Signs + symptoms of } \\
\text { spinal } \\
\text { metastasis/indication } \\
\text { for surgery }\end{array}$ & $\begin{array}{l}\text { Intervention for } \\
\text { spinal metastasis }\end{array}$ & $\begin{array}{l}\text { Outcome at last } \\
\text { follow-up } \\
\text { (months) }\end{array}$ \\
\hline \multirow[t]{8}{*}{ Quan et al., 2012} & 1 & $76, F$ & FWD & $\begin{array}{l}\text { T2 } \\
\text { No } \\
\text { M1 }\end{array}$ & \multirow{7}{*}{$\begin{array}{l}\text { Total thyroidectomy } \\
\text { followed by } \\
131-\text { ( } 100 \mathrm{mCi}) \\
\text { therapy every } \\
3-9 \text { months for } \\
2 \text { years then } \\
\text { once a year till } \\
\text { disappearance } \\
\text { of metastatic uptake }\end{array}$} & L3 & \multirow{4}{*}{$\begin{array}{l}\text { Intractable pain } \\
\text { resistant to } \\
\text { non-operative } \\
\text { measures and } \\
\text { pain and/or } \\
\text { paralysis due to } \\
\text { bony instability } \\
\text { or spinal cord } \\
\text { compression by } \\
\text { metastatic tumour }\end{array}$} & $\begin{array}{l}\text { L1-4 decompression } \\
\text { and stabilization }\end{array}$ & AWD (60) \\
\hline & 2 & $76, F$ & FWD & $\begin{array}{l}\text { T4 } \\
\text { No } \\
\text { M1 }\end{array}$ & & Lumbar & & $\begin{array}{l}\text { L4-5 decompression } \\
\text { and stabilization }\end{array}$ & AWD (56) \\
\hline & 3 & $60, F$ & PWD & $\begin{array}{l}\text { T1 } \\
\text { No } \\
\text { M1 }\end{array}$ & & $\llcorner 4$ & & $\begin{array}{l}\text { L3-5 decompression } \\
\text { and stabilization }\end{array}$ & AWD (20) \\
\hline & 4 & $67, F$ & FWD & $\begin{array}{l}\text { T4 } \\
\text { N1 } \\
\text { M1 }\end{array}$ & & Thoracic & & $\begin{array}{l}\text { T3 anterior corpectomy } \\
\text { and reconstruction } \\
\text { with cement and } \\
\text { anterior plate }\end{array}$ & CR (96) \\
\hline & 5 & $45, M$ & $\begin{array}{l}\text { Papillary PDTC, } \\
\text { refractory to I-131 }\end{array}$ & $\begin{array}{l}\text { T3 } \\
\text { NO } \\
\text { MO }\end{array}$ & & Thoracic & \multirow{4}{*}{$\begin{array}{l}\text { Intractable pain focal } \\
\text { mechanical pain } \\
\text { resistant to non-operative } \\
\text { measures and due } \\
\text { to osteolytic } \\
\text { metastases in the } \\
\text { absence of } \\
\text { significant spinal } \\
\text { cord compression } \\
\text { or spinal instability. }\end{array}$} & $\begin{array}{l}\text { T8 vertebroplasty, } \\
\text { followed by T8-T9 } \\
\text { decompression and } \\
\text { then T6, T7, and L3 } \\
\text { vertebroplasties }\end{array}$ & DOD (6) \\
\hline & 6 & $70, F$ & PWD & $\begin{array}{l}\text { T3 } \\
\text { N0 } \\
\text { M1 }\end{array}$ & & Thoracic & & $\begin{array}{l}\text { T9 and L1 } \\
\text { vertebroplasty }\end{array}$ & AWD (48) \\
\hline & 7 & $61, F$ & PWD & $\begin{array}{l}\text { T3 } \\
\text { N1 } \\
\text { M0 }\end{array}$ & & Cervical & & C3, non-operative & AWD (17) \\
\hline & 8 & $68, F$ & MTC & $\begin{array}{l}\text { T4 } \\
\text { No } \\
\text { M1 }\end{array}$ & Total thyroidectomy & Thoracic & & T12 vertebroplasty & DOD (18) \\
\hline \multirow[t]{4}{*}{$\begin{array}{l}\text { Demura et al., } \\
2011\end{array}$} & 1 & $55, F$ & Follicular & - & \multirow{4}{*}{$\begin{array}{l}7 / 10 \text { patients had } \\
\text { undergone } \\
\text { previous total } \\
\text { thyroidectomy. } \\
\text { Subtotal } \\
\text { thyroidectomy was } \\
\text { performed in } 2 \\
\text { patients. } \\
\text { Emergency spinal } \\
\text { surgery (TES) } \\
\text { was performed due } \\
\text { to acute paraparesis } \\
\text { in } 1 \text { case, } \\
\text { followed by total }\end{array}$} & L2 & $\begin{array}{l}\text { Preserved motor function, } \\
\text { abnormal gait and muscle } \\
\text { weakness }\end{array}$ & $\begin{array}{l}\text { SET, TES \& } \\
\text { intraoperative } \\
\text { chemotherapy }\end{array}$ & DOD (77) \\
\hline & 2 & $56, F$ & Follicular & - & & $\mathrm{T} 7$ & $\begin{array}{l}\text { Preserved motor function } \\
\text { and normal gait }\end{array}$ & $\begin{array}{l}\text { SET, TES \& } \\
\text { intraoperative } \\
\text { chemotherapy }\end{array}$ & NED (125) \\
\hline & 3 & $58, F$ & Papillary & - & & $\mathrm{T} 2$ & $\begin{array}{l}\text { Preserved motor, } \\
\text { non-functional }\end{array}$ & $\begin{array}{l}\text { SET, TES \& } \\
\text { intraoperative } \\
\text { chemotherapy }\end{array}$ & DOD (27) \\
\hline & 4 & $56, F$ & Follicular & - & & L2 & Normal motor function & $\begin{array}{l}\text { SET, TES \& } \\
\text { intraoperative } \\
\text { chemotherapy }\end{array}$ & AWD (86) \\
\hline
\end{tabular}


Table 2 Clinical characteristics at diagnosis of patients with spinal metastasis from thyroid carcinoma and the therapeutic strategies deployed (Continued)

\begin{tabular}{|c|c|c|c|c|c|c|c|c|c|}
\hline & 5 & $59, \mathrm{M}$ & Papillary & - & $\begin{array}{l}\text { thyroidectomy. } \\
\text { Preoperatively, 131-I } \\
\text { therapy had }\end{array}$ & $\mathrm{T} 10$ & Normal motor function & $\begin{array}{l}\text { SET, TES \& } \\
\text { intraoperative } \\
\text { chemotherapy }\end{array}$ & DOD (62) \\
\hline & 6 & $63, F$ & Follicular & - & $\begin{array}{l}\text { been administered } \\
\text { in } 4 \text { cases } \\
\text { (patient 2, 3, 5, } \\
\text { and 9); }\end{array}$ & T10-11 & $\begin{array}{l}\text { Preserved motor, } \\
\text { non-functional }\end{array}$ & $\begin{array}{l}\text { SET, TES \& } \\
\text { intraoperative } \\
\text { chemotherapy }\end{array}$ & AWD (56) \\
\hline & 7 & $39, F$ & Medullary & - & $\begin{array}{l}\text { all } 4 \text { of these } \\
\text { patients showed a } \\
\text { poor response to }\end{array}$ & $\mathrm{T} 2, \mathrm{~T} 5-7$ & Normal & $\begin{array}{l}\text { SET, TES \& } \\
\text { intraoperative } \\
\text { chemotherapy }\end{array}$ & AWD (52) \\
\hline & 8 & $77, \mathrm{~F}$ & Follicular & - & the treatment. & $\mathrm{T} 7, \mathrm{~T} 10$ & $\begin{array}{l}\text { Preserved } \\
\text { motor function, } \\
\text { abnormal gait } \\
\text { and muscle } \\
\text { weakness and } \\
\text { bladder paralysis }\end{array}$ & $\begin{array}{l}\text { SET, TES \& } \\
\text { intraoperative } \\
\text { chemotherapy }\end{array}$ & AWD (45) \\
\hline & 9 & $57, \mathrm{~F}$ & Follicular & - & & $\mathrm{T} 11$ & $\begin{array}{l}\text { Normal motor } \\
\text { function }\end{array}$ & $\begin{array}{l}\text { SET, TES \& } \\
\text { intraoperative } \\
\text { chemotherapy }\end{array}$ & AWD (36) \\
\hline & 10 & $52, F$ & Papillary & - & & $\mathrm{L} 2-4$ & $\begin{array}{l}\text { Normal motor } \\
\text { function }\end{array}$ & $\begin{array}{l}\text { SET, TES \& } \\
\text { intraoperative } \\
\text { chemotherapy }\end{array}$ & NED (36) \\
\hline $\begin{array}{l}\text { De Vries et al., } \\
2008\end{array}$ & 1 & $60, F$ & FWD & $\begin{array}{l}\text { T1 } \\
\text { No } \\
\text { M1 }\end{array}$ & $\begin{array}{l}\text { Total thyroidectomy } \\
\text { and I-131 therapy }\end{array}$ & L4 & $\begin{array}{l}\text { Pain and } \\
\text { neurological } \\
\text { symptoms }\end{array}$ & $\begin{array}{l}\text { SET, external beam } \\
\text { radiotherapy \& } \\
\text { corporectomy } L 4\end{array}$ & $\begin{array}{l}\text { Symptoms decreased } \\
\text { during } 17 \text { sessions } \\
\text { (mean duration of }\end{array}$ \\
\hline & 2 & $62, F$ & FWD & $\begin{array}{l}\text { T2 } \\
\text { NO } \\
\text { M1 }\end{array}$ & & $\mathrm{T} 5-7$ & $\begin{array}{l}\text { Pain and } \\
\text { neurological } \\
\text { symptoms }\end{array}$ & $\begin{array}{l}\text { SET \& external beam } \\
\text { radiotherapy }\end{array}$ & $\begin{array}{l}8.1 \text { months), were } \\
\text { unchanged } \\
\text { during } 8 \text { sessions, } \\
\text { and increased }\end{array}$ \\
\hline & 3 & $65, F$ & FWD & $\begin{array}{l}\text { T2 } \\
\text { N0 } \\
\text { M1 }\end{array}$ & & L3- 4 & $\begin{array}{l}\text { Pain and } \\
\text { neurological } \\
\text { symptoms }\end{array}$ & SET & $\begin{array}{l}\text { during } 6 \text { sessions } \\
(p<0.01) .\end{array}$ \\
\hline & 4 & $40, F$ & FWD & $\begin{array}{l}\text { T4 } \\
\text { N0 } \\
\text { M1 }\end{array}$ & & L5 & $\begin{array}{l}\text { Pain and } \\
\text { neurological } \\
\text { symptoms }\end{array}$ & $\begin{array}{l}\text { SET \& external beam } \\
\text { radiotherapy }\end{array}$ & \\
\hline & 5 & $77, \mathrm{~F}$ & FWD & $\begin{array}{l}\text { T2 } \\
\text { N0 } \\
\text { M1 }\end{array}$ & & L2 & $\begin{array}{l}\text { Pain and } \\
\text { neurological } \\
\text { symptoms }\end{array}$ & $\begin{array}{l}\text { SET \& external beam } \\
\text { radiotherapy }\end{array}$ & \\
\hline & 6 & $54, F$ & FWD & $\begin{array}{l}\text { T2 } \\
\text { NO } \\
\text { MO }\end{array}$ & & $\mathrm{T} 10-11$ & $\begin{array}{l}\text { Pain and } \\
\text { neurological } \\
\text { symptoms }\end{array}$ & $\begin{array}{l}\text { SET \& external beam } \\
\text { radiotherapy }\end{array}$ & \\
\hline & 7 & $35, M$ & FWD & $\begin{array}{l}\text { Tx } \\
\text { NO } \\
\text { MO }\end{array}$ & & $\mathrm{L} 2-4$ & $\begin{array}{l}\text { Pain and } \\
\text { neurological } \\
\text { symptoms }\end{array}$ & $\begin{array}{l}\text { SET \& external beam } \\
\text { radiotherapy }\end{array}$ & \\
\hline
\end{tabular}


Table 2 Clinical characteristics at diagnosis of patients with spinal metastasis from thyroid carcinoma and the therapeutic strategies deployed (Continued)

\begin{tabular}{|c|c|c|c|c|c|c|c|c|c|}
\hline & 8 & $52, F$ & FWD & $\begin{array}{l}\text { Tx } \\
\text { N0 } \\
\text { M1 }\end{array}$ & & L3 & $\begin{array}{l}\text { Pain and } \\
\text { neurological } \\
\text { symptoms }\end{array}$ & SET \& corporectomy L3 & \\
\hline & 9 & $45, F$ & FWD & $\begin{array}{l}\text { T3 } \\
\text { N0 } \\
\text { M1 }\end{array}$ & & C4 & $\begin{array}{l}\text { Pain and } \\
\text { neurological } \\
\text { symptoms }\end{array}$ & SET \& corporectomy C4 & \\
\hline & 10 & $83, \mathrm{M}$ & FWD & $\begin{array}{l}\text { Tx } \\
\text { N0 } \\
\text { M1 }\end{array}$ & & C5-6 & $\begin{array}{l}\text { Pain and } \\
\text { neurological } \\
\text { symptoms }\end{array}$ & SET \& surgical C5-C6 & \\
\hline \multirow[t]{9}{*}{$\begin{array}{l}\text { Hindie í et al., } \\
2007\end{array}$} & 1 & $27, F$ & $\begin{array}{l}\text { FWD } \\
\text { (Hurthle areas) }\end{array}$ & $\begin{array}{l}\text { T2 } \\
\text { NX }\end{array}$ & \multirow{9}{*}{$\begin{array}{l}\text { Total thyroidectomy } \\
\text { and } 131-I \text { ablation } \\
\text { therapy }(100 \mathrm{mCi})\end{array}$} & $\mathrm{T} 2$ & Back pain & $\begin{array}{l}\text { Spinal surgery+ } \\
\text { radiotherapy }\end{array}$ & PR (43) \\
\hline & 2 & $34, F$ & PWD & $\begin{array}{l}\text { T3 } \\
\text { NX }\end{array}$ & & $\llcorner 4$ & $\begin{array}{l}\text { Asymptomatic, } \\
\text { metastases revealed } \\
\text { via } 131-I \text { ablation therapy }\end{array}$ & - & CR (8) \\
\hline & 3 & $48, M$ & PWD & $\begin{array}{l}\text { T1 } \\
\text { NX }\end{array}$ & & T6, L2 & $\begin{array}{l}\text { Asymptomatic, } \\
\text { metastases revealed } \\
\text { via } 131-\text { ablation therapy }\end{array}$ & - & CR (37) \\
\hline & 4 & $53, \mathrm{M}$ & Follicular, PDTC & $\begin{array}{l}\text { T4a } \\
\text { N1b }\end{array}$ & & T6 & Back pain & $\begin{array}{l}\text { Spinal surgery }+ \\
\text { radiotherapy }\end{array}$ & DOD (76) \\
\hline & 5 & $55, \mathrm{M}$ & $\begin{array}{l}\text { PWD } \\
\text { (Hurthle areas) }\end{array}$ & $\begin{array}{l}\mathrm{T} 1 \\
\mathrm{Nx}\end{array}$ & & T11, L3 & $\begin{array}{l}\text { Asymptomatic, } \\
\text { metastases revealed } \\
\text { via } 131-I \text { ablation therapy }\end{array}$ & - & CR (23) \\
\hline & 6 & $63, F$ & FWD & $\begin{array}{l}\mathrm{T} 2 \\
\mathrm{NX}\end{array}$ & & T10-11 & Back pain & Spine radiotherapy & DOD (32) \\
\hline & 7 & $75, F$ & FWD & $\begin{array}{l}\text { T3 } \\
\text { NO }\end{array}$ & & $\mathrm{T} 12$ & Back pain & $\begin{array}{l}\text { Spinal surgery + } \\
\text { radiotherapy }\end{array}$ & PD (62) \\
\hline & 8 & $65, F$ & PWD & $\begin{array}{l}\text { T4b } \\
\text { N1b }\end{array}$ & & C6 & $\begin{array}{l}\text { Asymptomatic, } \\
\text { metastases } \\
\text { revealed } \\
\text { via 131-I ablation } \\
\text { therapy }\end{array}$ & Spine radiotherapy & DOD (34) \\
\hline & 9 & $73, \mathrm{M}$ & Hürthle & $\begin{array}{l}\text { T3 } \\
\text { N1b }\end{array}$ & & Cervical & Bone pain & Spine radiotherapy & DOD (5) \\
\hline \multirow[t]{3}{*}{$\begin{array}{l}\text { Eustatia-Rutten et al., } \\
2003\end{array}$} & 1 & $69, M$ & FTC, DTC & $\begin{array}{l}\text { T4 } \\
\text { N1 } \\
\text { M1 }\end{array}$ & \multirow{3}{*}{$\begin{array}{l}\text { Total thyroidectomy } \\
\text { and } 131-\text { ablation } \\
\text { therapy }(76-162 \mathrm{mCi}) \pm \\
\text { second } 131-\text { I therapy, } \\
\text { irradiation and/or } \\
\text { laminectomy }\end{array}$} & L1-4 & $\begin{array}{l}\text { Radicular pain/SSC, } \\
\text { hyperaesthesia }\end{array}$ & $\begin{array}{l}\text { SET and } 131-I \text { ablation } \\
\text { therapy }(76-162 \mathrm{mCi}) \text {, } \\
\text { laminectomy and } \\
\text { irradiation }\end{array}$ & DOD (54) \\
\hline & 2 & $51, M$ & FTC & $\begin{array}{l}\text { Tx } \\
\mathrm{Nx} \\
\mathrm{MO}\end{array}$ & & $\mathrm{T} 1$ & Radicular pain & SET & DOD (124) \\
\hline & 3 & $72, F$ & PTC & $\begin{array}{l}\text { T4 } \\
\text { N0 } \\
\text { M1 }\end{array}$ & & $\mathrm{T} 1$ & Paresis, incontinence & $\begin{array}{l}\text { SET then } \\
\text { laminectomy } \\
\& \text { irradiation }\end{array}$ & DOD (1.5) \\
\hline
\end{tabular}


Table 2 Clinical characteristics at diagnosis of patients with spinal metastasis from thyroid carcinoma and the therapeutic strategies deployed (Continued)

\begin{tabular}{|c|c|c|c|c|c|c|c|c|c|}
\hline & 4 & $71, F$ & PTC & $\begin{array}{l}\text { T4 } \\
\text { N0 } \\
\text { M1 }\end{array}$ & & L3 & Radicular pain/SSC, & SET then irradiation & PD (139) \\
\hline & 5 & $62, \mathrm{M}$ & FTC & $\begin{array}{l}\text { T4 } \\
\text { NO } \\
\text { MO }\end{array}$ & & $\begin{array}{l}\text { T10, } \\
\text { T12 }\end{array}$ & Radicular pain & SET & DOD (63) \\
\hline & 6 & $60, M$ & FTC & $\begin{array}{l}\text { T2 } \\
\text { N0 } \\
\text { M1 }\end{array}$ & & $C 7$ & $\begin{array}{l}\text { SSC, paraplegia, } \\
\text { radicular pain }\end{array}$ & $\begin{array}{l}\text { SET then 131-I therapy } \\
\text { (76-162 mCi) } \\
\text { and irradiation }\end{array}$ & DOD (86) \\
\hline & 7 & $53, F$ & FTC & $\begin{array}{l}\text { T2 } \\
\text { NO } \\
\text { MO }\end{array}$ & & L1 & $\begin{array}{l}\text { Radicular pain, } \\
\text { hyperaesthesia, } \\
\text { paraesthesia }\end{array}$ & SET & PD (108) \\
\hline & 8 & $45, M$ & FTC & $\begin{array}{l}\text { T3 } \\
\text { NO } \\
\text { M0 }\end{array}$ & & $\begin{array}{l}\text { T1-3, } \\
\text { T5-7 }\end{array}$ & $\begin{array}{l}\text { Radicular pain, } \\
\text { paraparesis, } \\
\text { ataxia, paraplegia }\end{array}$ & $\begin{array}{l}\text { SET then } 131-1 \text { ablation } \\
\text { therapy }(76-162 \mathrm{mCi}) \text {, } \\
\text { laminectomy and irradiation }\end{array}$ & PD (152) \\
\hline & 9 & $72, \mathrm{M}$ & PTC & $\begin{array}{l}\text { Tx } \\
\text { N1 } \\
\text { M1 }\end{array}$ & & T9-10 & Radicular pain, & SET & DOD (12) \\
\hline & 10 & $62, \mathrm{M}$ & PTC & $\begin{array}{l}\text { T3 } \\
\text { NO } \\
\text { MO }\end{array}$ & & T9-10 & $\begin{array}{l}\text { paraparesis, } \\
\text { incontinence, } \\
\text { hyperaesthesia, } \\
\text { paraplegia, } \\
\text { radicular pain }\end{array}$ & SET & DOD (102) \\
\hline \multirow[t]{3}{*}{$\begin{array}{l}\text { van Tol et al., } \\
2000\end{array}$} & 1 & $77, F$ & PWD & $\begin{array}{l}\mathrm{T} 2 \\
\mathrm{~N} 1 \\
\mathrm{M} 1\end{array}$ & \multirow{3}{*}{$\begin{array}{l}\text { Total thyroidectomy } \\
\text { and } 131-\text { I } \\
\text { ablation therapy } \\
(150 \mathrm{mCi})\end{array}$} & L2 & $\begin{array}{l}\text { Severe pain and } \\
\text { neurological } \\
\text { symptoms }\end{array}$ & $\begin{array}{l}\text { SET then } 131-1 \\
\text { therapy }(150 \mathrm{mCi})\end{array}$ & AWD (40) \\
\hline & 3 & $63, F$ & FWD & $\begin{array}{l}\text { T2 } \\
\text { No } \\
\text { M1 }\end{array}$ & & $\mathrm{T7}$ & $\begin{array}{l}\text { Severe pain, neurological } \\
\text { symptoms and SCC }\end{array}$ & $\begin{array}{l}\text { SET then } 131-1 \\
\text { therapy }(150 \mathrm{mCi})\end{array}$ & CR (38) \\
\hline & 4 & $60, F$ & FWD & $\begin{array}{l}\text { T2 } \\
\text { N0 } \\
\text { M1 }\end{array}$ & & $\llcorner 4$ & $\begin{array}{l}\text { Severe pain, neurological } \\
\text { symptoms and SCC }\end{array}$ & $\begin{array}{l}\text { SET then 131-I } \\
\text { therapy (150 mCi) }\end{array}$ & CR (21) \\
\hline \multirow[t]{4}{*}{$\begin{array}{l}\text { Vitale et al. } \\
2001\end{array}$} & 1 & $35, F$ & FWD & - & \multirow{4}{*}{$\begin{array}{l}\text { Not specified; } \\
\text { failure of all } \\
\text { conventional } \\
\text { therapy }\end{array}$} & Spine & Painful bone metastases & $\begin{array}{l}1 \text { year monthly course } \\
\text { of I.V. pamidronate } \\
\text { ( } 90 \mathrm{mg} \text { ) }\end{array}$ & PR (12) \\
\hline & 2 & $76, F$ & PWD & - & & Spine & Painful bone metastases & $\begin{array}{l}1 \text { year monthly course } \\
\text { of I.V. pamidronate } \\
(90 \mathrm{mg})\end{array}$ & AWD (12) \\
\hline & 3 & $48, F$ & FWD & - & & Spine & Painful bone metastases & $\begin{array}{l}1 \text { year monthly course } \\
\text { of I.V. pamidronate } \\
(90 \mathrm{mg})\end{array}$ & PR (12) \\
\hline & 4 & $46, F$ & FWD & - & & Spine & Painful bone metastases & $\begin{array}{l}1 \text { year monthly course } \\
\text { of I.V. pamidronate } \\
(90 \mathrm{mg})\end{array}$ & AWD (12) \\
\hline
\end{tabular}


Table 2 Clinical characteristics at diagnosis of patients with spinal metastasis from thyroid carcinoma and the therapeutic strategies deployed (Continued)

\begin{tabular}{|c|c|c|c|c|c|c|c|}
\hline 5 & $54, F$ & MTC & - & Spine & Painful bone metastases & $\begin{array}{l}1 \text { year monthly course } \\
\text { of I.V. pamidronate } \\
(90 \mathrm{mg})\end{array}$ & $\mathrm{PD}(12)$ \\
\hline 6 & $66, F$ & MTC & - & Spine & Painful bone metastases & $\begin{array}{l}7 \text { monthly course of } \\
\text { I.V. pamidronate } \\
(90 \mathrm{mg})\end{array}$ & PD (12) \\
\hline 7 & $61, M$ & FWD & - & Spine & Painful bone metastases & $\begin{array}{l}1 \text { year monthly course } \\
\text { of I.V. pamidronate } \\
\text { ( } 90 \mathrm{mg} \text { ) }\end{array}$ & AWD (12) \\
\hline \multicolumn{8}{|c|}{$\begin{array}{l}\text { DTC; differentiated thyroid carcinoma, PWD; papillary well differentiated, FWD; follicular well differentiated, MTC; medullary thyroid carcinoma, PDTC; poorly differentiated thyroid carcinoma, FLD; follicular less } \\
\text { differentiated, UTC; undifferentiated thyroid carcinoma, T; thoracic, L; lumbar, C; cervical, BM; bone metastasis, SM; spinal metastasis, SSC; spinal cord compression, RAI; radioactive iodine (131-I), ERT; external } \\
\text { radiotherapy, CT; chemotherapy, SET; selective embolization therapy, TES; total en bloc spondylectomy, ZA; zolendronic acid, NED; no evidence of disease, SD; stable disease, CR; complete remission, PR; partia } \\
\text { response, PD; progressive disease, AWD; alive with disease, DOD; death of disease, SRE; skeletal-related events, PFS; progression-free survival, DSS; disease-specific survival, Yrs; years. }\end{array}$} \\
\hline
\end{tabular}


Table 3 Summary results of studies that deployed conventional therapeutic modalities in patients with distant metastasis from thyroid carcinoma

\begin{tabular}{|c|c|c|c|c|c|c|c|c|}
\hline Author, Year & $\begin{array}{l}\text { Number of } \\
\text { patients } \\
\text { (\% female) }\end{array}$ & $\begin{array}{l}\text { Median age (yrs) } \\
\text { at time of } \\
\text { symptomatic } \\
\text { distant metastatic } \\
\text { disease (range) }\end{array}$ & $\begin{array}{l}\text { Thyroid cancer } \\
\text { histology (\%) }\end{array}$ & $\begin{array}{l}\text { Percentage of } \\
\text { Patients with } \\
\text { bone/spinal } \\
\text { metastasis }\end{array}$ & $\begin{array}{l}\text { Signs and symptoms } \\
\text { of distant metastasis/ } \\
\text { indication for } \\
\text { intervention }\end{array}$ & $\begin{array}{l}\text { Intervention for } \\
\text { distant metastasis } \\
\text { (\% of patients) }\end{array}$ & $\begin{array}{l}\text { Other } \\
\text { metastases } \\
(\%)\end{array}$ & Main outcome(s) \\
\hline $\begin{array}{l}\text { Schlumberger } \\
\text { et al., } 1999\end{array}$ & $394(61.7 \%)$ & - & $\begin{array}{l}\text { PWD (46.1\%), } \\
\text { FWD (7.5\%), } \\
\text { FLD (46.3\%) }\end{array}$ & $\begin{array}{l}\text { BM (27.4\%), } \\
\text { BM + Lung } \\
(18.3 \%)\end{array}$ & $\begin{array}{l}\text { Pain, swelling, } \\
\text { orthopaedic } \\
\text { complications }\end{array}$ & $\begin{array}{l}\text { RAI (88\%), } \\
\text { ERT (35\%), } \\
\text { surgery (18\%), } \\
\text { CT }(12 \%)\end{array}$ & Lung (54.3\%) & $\begin{array}{l}5 \text { year/10 year/ } \\
15 \text { year survival: } \\
55 \% / 40 \% / 33 \%\end{array}$ \\
\hline $\begin{array}{l}\text { Pittas et al. } \\
2000\end{array}$ & $146(58 \%)$ & 58.7 & $\begin{array}{l}\text { PWD (13\%), } \\
\text { FWD (12\%), } \\
\text { Hurthle (6\%), } \\
\text { UTC (7), } \\
\text { PDTC (13\%), } \\
\text { medullary (4\%), } \\
\text { lymphoma (2\%), } \\
\text { unknown (43\%) }\end{array}$ & $\begin{array}{l}\text { BM (100\%), } \\
\text { SM (54\%) }\end{array}$ & $\begin{array}{l}\text { Pain }(50 \%), \\
\text { swelling (11.0\%), } \\
\text { pathologic fracture } \\
(4.1 \%), \text { SCC }(3.4 \%)\end{array}$ & $\begin{array}{l}\text { RAI }(51 \%), \\
\text { ERT }(75 \%), \\
\text { Surgery (26\%), } \\
\text { CT }(12 \%)\end{array}$ & Other (36\%) & $\begin{array}{l}5 \mathrm{yr} / 10 \text { yr survival: } \\
53 \% / 35 \%\end{array}$ \\
\hline $\begin{array}{l}\text { Bernier et al., } \\
2001\end{array}$ & 109 (71\%) & $63(20-87)$ & $\begin{array}{l}\text { PWD (17\%), } \\
\text { FWD (71\%), } \\
\text { Unknown (12\%) }\end{array}$ & $\begin{array}{l}\text { BM (100\%), } \\
\text { SM (68\%) }\end{array}$ & $\begin{array}{l}\text { Pathological } \\
\text { fractures (13\%), } \\
\text { Radiculalgia (4\%), } \\
\text { SCC (28\%), } \\
\text { SCC + fracture } \\
(6 \% \text { r }\end{array}$ & $\begin{array}{l}\text { Complete BM } \\
\text { surgery (22\%), } \\
\text { palliative } \\
\text { surgery }(55 \%), \\
\text { ERT }(36 \%), \\
\text { SET }(31 \%), \\
\text { CT }(2 \%)\end{array}$ & $\begin{array}{l}\text { Lung }(34 \%), \\
\text { thyroid (11.\%), } \\
\text { other (25\%) }\end{array}$ & $\begin{array}{l}5 \mathrm{yr} / 10 \mathrm{yr} / 20 \mathrm{yr} \\
\text { survival rate: } \\
41 \% / 15 \% / 7 \% \text {, Mean } \\
\text { survival } 5.6 \text { years. } \\
\text { Remission rate } 4 \% \text {, } \\
\text { Mortality } 84 \%\end{array}$ \\
\hline $\begin{array}{l}\text { Petrich et al., } \\
2001\end{array}$ & 107 (28.0\%) & 62.1 & $\begin{array}{l}\text { PWD (27.1\%), } \\
\text { FWD (72.9\%) }\end{array}$ & BM (100\%) & - & $\begin{array}{l}\text { RAI (100\%), } \\
\text { ERT (14\%) }\end{array}$ & Lung (41.1\%) & $\begin{array}{l}\text { CR } 25 \text { (23.4\%), } \\
\text { PR } 29(27.1 \%), \\
\text { PD } 53(49.5 \%), \\
\text { Mean survival } 7.9 \mathrm{yr}\end{array}$ \\
\hline $\begin{array}{l}\text { Stojadinovic et al., } \\
2002\end{array}$ & $260(51.2 \%)$ & $58(5-91)$ & $\begin{array}{l}\text { PWD (58.8\%), } \\
\text { FWD (34.2\%), } \\
\text { Hurthle (6.9\%) }\end{array}$ & BM (15\%) & - & $\begin{array}{l}\text { Surgery (22.7\%), + } \\
\text { RAI (13.8\%), RAI +/- } \\
\text { Other (54.2\%), } \\
\text { Other (ERT, CT, } \\
\text { supportive) (23.1\%) }\end{array}$ & $\begin{array}{l}\text { Lung (32.7\%), } \\
\text { single site } \\
59(22.7 \%) \\
\text { multisite } \\
77(29.6 \%)\end{array}$ & $\begin{array}{l}5 \text { yr DSS: Complete } \\
\text { metastasectomy 78\%, } \\
\text { Partial resection 43\%, } \\
\text { Non-operative } \\
\text { treatment 46\% }\end{array}$ \\
\hline $\begin{array}{l}\text { Zettinig et al., } \\
2002\end{array}$ & $41(58.5 \%)$ & $60+/-12$ & $\begin{array}{l}\text { PWD (14.6\%), } \\
\text { FWD (85.4\%) }\end{array}$ & BM (100\%) & $\begin{array}{l}\text { Pain (37\%), } \\
\text { goitre/nodules (51\%), } \\
\text { dyspnoea (10\%) }\end{array}$ & $\begin{array}{l}\text { Surgery (51.2\%), } \\
\text { RAI (78\%), } \\
\text { ERT (27\%), CT (6\%) }\end{array}$ & - & $\begin{array}{l}5 \mathrm{yr} / 10 \mathrm{yr} \text { survival: } \\
69.2 \% / 38.9 \%\end{array}$ \\
\hline $\begin{array}{l}\text { Durante et al., } \\
2006\end{array}$ & $444(62 \%)$ & - & $\begin{array}{l}\text { PWD (42\%), } \\
\text { FWD (15\%), } \\
\text { FPD (41\%) }\end{array}$ & $\begin{array}{l}\text { BM (26\%), } \\
\text { BM and lung } \\
(18 \%)\end{array}$ & - & $\begin{array}{l}\mathrm{RAI}+\mathrm{CT} \text {, EBR } \\
\text { and/or surgery } \\
\text { in indicated } \\
\text { BM patients }\end{array}$ & $\begin{array}{l}\text { Lung }(52 \%) \text {, } \\
\text { other }(5 \%)\end{array}$ & $\begin{array}{l}10 \text { yr survival in } \\
\text { negative and } \\
\text { abnormal study } \\
\text { patients, } 92 \% \text { and } 19 \% \text {, } \\
\text { respectively. } \\
\text { BM only patients required } \\
\text { median cumulative } 1-131 \\
\text { dose of } 250 \text { mCi to } \\
\text { attain negative studies }\end{array}$ \\
\hline Orita et al., 2010 & $52(65.4 \%)$ & $59(32-77)$ & $\begin{array}{l}\text { PWD (52\%), } \\
\text { FWD (48.\%) }\end{array}$ & BM (100\%) & $\begin{array}{l}\text { Pain }(46.2 \%) \text {, mass bone } \\
\text { lesions }(9.6 \%) \text {, }\end{array}$ & $\begin{array}{l}\text { Surgery (21.2\%), } \\
\text { ERT }(75 \%), \\
\text { RAI }(75 \%),\end{array}$ & $\begin{array}{l}\text { Lung }(44.2 \%), \\
\text { pleura }(9.6 \%),\end{array}$ & 5 yr/10 yr DSS: $36 \% / 10 \%$ \\
\hline
\end{tabular}


Table 3 Summary results of studies that deployed conventional therapeutic modalities in patients with distant metastasis from thyroid carcinoma (Continued)

\begin{tabular}{|c|c|c|c|c|c|c|c|c|}
\hline \multirow[b]{2}{*}{ Qiu et al., 2011} & \multirow[b]{2}{*}{$106(62 \%)$} & \multirow[b]{2}{*}{$53(12-85)$} & \multirow[b]{2}{*}{$\begin{array}{l}\text { PWD (41.5\%), } \\
\text { FWD (49.1\%), } \\
\text { Follicular variant } \\
\text { of Papillary (9.4\%) }\end{array}$} & \multirow[b]{2}{*}{$\begin{array}{l}\text { BM (45.3\%), } \\
\text { BM + other } \\
(54.7 \%)\end{array}$} & \multirow{2}{*}{$\begin{array}{l}\text { paralysis/numbness (7.7\%), } \\
\text { fracture (3.8\%) } \\
\text { Pain }(57.5 \%), \text { pathological } \\
\text { fracture (5.7\%), SCC (4.7\%) }\end{array}$} & \multirow{2}{*}{$\begin{array}{l}\text { CT (7.7\%), } \\
\text { Bisphosphonate (34.6\%), } \\
\text { TSH suppression (36.5\%) } \\
\text { Oral RAI (100\%), } \\
\text { Surgery }(24.5 \%)\end{array}$} & \multicolumn{2}{|l|}{$\begin{array}{l}\text { brain }(5.8 \%) \text {, } \\
\text { liver }(3.8 \%)\end{array}$} \\
\hline & & & & & & & $\begin{array}{l}\text { Cervical lymph } \\
\text { nodes }(16.0 \%) \text {, } \\
\text { lung }(42 . \%), \\
\text { other }(20 \%)\end{array}$ & $\begin{array}{l}5 \mathrm{yr} / 10 \mathrm{yr} \text { survival rate: } \\
86.5 \% / 57.9 \%\end{array}$ \\
\hline \multicolumn{9}{|c|}{$\begin{array}{l}\text { DTC; differentiated thyroid carcinoma, PWD; papillary well differentiated, FWD; follicular well differentiated, MTC; medullary thyroid carcinoma, PDTC; poorly differentiated thyroid carcinoma, FLD; follicular less } \\
\text { differentiated, UTC; undifferentiated thyroid carcinoma, T; thoracic, L; lumbar, C; cervical, BM; bone metastasis, SM; spinal metastasis, SSC; spinal cord compression, RAI; radioactive iodine (131-I), ERT; external } \\
\text { radiotherapy, CT; chemotherapy, SET; selective embolization therapy, TES; total en bloc spondylectomy, ZA; zolendronic acid, NED; no evidence of disease, SD; stable disease, CR; complete remission, PR; partial } \\
\text { response, PD; progressive disease, AWD; alive with disease, DOD; death of disease, SRE; skeletal-related events, PFS; progression-free survival, DSS; disease-specific survival, yr; year. }\end{array}$} \\
\hline
\end{tabular}


of the vertebral body compromises the supportive structure of the spine [40]. Compression of the cord or cauda equina induces severe pain, paralysis and sphincter dysfunction [40,48]. Therapeutic interventions should, therefore, target first and foremost the integrity of the spine to prevent neurological complications. In doing so, they may vouchsafe symptomatic control, such as pain, paralysis and the impact these have on activities of daily living [48].

Once spinal metastasis has been diagnosed, there are a several modalities of treatment. Treatment can be palliative or curative. The use of radioiodine ablation therapy, selective embolisation therapy (SET), bisphosphonates, surgery, and small molecular therapy is being discussed [40].

Approximately $90 \%$ of patients with spinal metastasis experience pain [25]. The pain can be significantly debilitating and interfere with quality of life. Pain, in metastatic cancer, is managed by a multidisciplinary team; treatments include analgesia, bisphosphonates, radiotherapy and chemotherapy [50]. In terms of spinal metastasis, intractable pain is often a surgical indication [13], and many other treatments such as SET and radioiodine have been shown to reduce pain scores [44].

\section{Radioiodine ablation therapy}

Radioiodine therapy is a mainstay of thyroid cancer management [10,19,31]. Many studies reported benefit retrospectively. Intriguingly, Van Tol et al. [44] demonstrated that radioiodine ablation reduces the pain rating on a 4 point analogue scale, irrespective of whether SET was combined, in a number of patients with spinal metastasis. Radioiodine absorption is a prognostic factor in metastatic disease [14]. Tumours, which are nonfunctioning, represent an entity further down the pathway of malignant transformation and they are resistant to the radiation dose delivered through radioiodine sequestration. Hindie et al. recommend that young patients who have ${ }^{131} \mathrm{I}$ avid metastasis, of a papillary or follicular, well differentiated nature, should receive ${ }^{131} \mathrm{I}$. Durante et al. [30] observed that once a patient achieved a positive scan, it on average took a cumulative dose of up to $600 \mathrm{mCi}$ to achieve a negative scan. It has been shown that a dose exceeding this value can lead to increased risk of haematological malignancy, and salivary gland dysfunction. They suggested, therefore, that if patients have been treated with $600 \mathrm{mCi}$, as a cumulative dose, deciding to re-treat should be made on an individual basis.

\section{Selective embolisation therapy}

SET represents an attractive therapy due to its induction of rapid, albeit, transient symptom amelioration. Eustatia Rutten et al. [16] suggested that symptomatic control and progression arrest is achievable in 59\% of embolizations. In reality, however, this does not equate to $59 \%$ of patients receiving neurological and pain benefit. They also found that the benefit lasted a mean duration of 6.5 months suggesting that more sessions may be required to continue to ameliorate symptoms [16]. There is evidence, albeit sparse, of its efficacy in cervical instability $[16,36]$. Serum thyroglobulin $(\mathrm{Tg})$ a marker of tumour burden, was radically reduced in an a study by Van Tol et al. in the embolisation group, (88.7\% c.f. $18.6 \%$ in the control; $\mathrm{p}<0.05)$. Of course, this is only a proxy for disease. De Vries et al. [36] postulated that embolisation may be beneficial in not only palliating symptoms, but can be used in an adjuvant setting, a form of pre-operative embolisation to help reduce blood loss ${ }^{37}$. Unfortunately, SET does not appear to improve survival $[16,36]$. SET alone induces tissue hypoxia, a stimulus for tumour growth. The combination of SET with radiation therapy may confer a synergistic benefit.

\section{Surgery}

The role of surgery is debatable. Quan et al. [40] suggests that surgery is indicated for patients with intractable pain, cord compression, neurological deficit or cervical instability [40]. Cervical metastasis can produce pain unresponsive to medical management, which can affect activities of daily living. In addition neurological deficits and pathological fractures can occur due to metastatic lesions reducing the integrity of the vertebral bodies [50]. Metastatic cervical spine disease causing instability is usually treated with anterior reconstruction and stabilisation [51] Bernier et al. [34] reported that surgery as a whole did not improve survival, but complete bone metastasis (BM) resection did [34]. A factor for deciding which type of surgery patients were given was age. Surgical treatment in the young should, therefore, be more aggressive. Demura et al. [13] suggested that total en bloc spondylectomy (TES) may provide better local control of thyroid cancer spinal metastasis, compared with debulking surgery. Complete resection of synchronous metastasis was associated with increased survival [13]. The authors showed a significant difference in the risk of local recurrence with debulking surgery (risk of recurrence $57 \%$, c.f. $10 \%$ with TES). Patients who underwent complete metastasectomy had significantly improved survival than those having palliative resection (5-year DSS, $70 \%$ vs. $30 \%, P$ value $=0.004$ ) [43]. This indicates, perhaps, that it is important to treat as many metastasis burden as possible, however, this may not always be achievable. Although, there is considerable bias in this conclusion, in that those patients whose disease is too extensive to treatment would, of course, have a poorer survival. Kushchayev et al. [23] 
Table 4 Summary results of studies that deployed novel therapeutic modalities in patients with distant metastases from thyroid carcinomas

\begin{tabular}{|c|c|c|c|c|c|c|c|c|}
\hline Author, Year & $\begin{array}{l}\text { Number of } \\
\text { patients } \\
\text { (\% female) }\end{array}$ & $\begin{array}{l}\text { Median age (yrs) } \\
\text { at time of } \\
\text { symptomatic } \\
\text { distant } \\
\text { metastatic disease } \\
\text { (range) }\end{array}$ & $\begin{array}{l}\text { Thyroid cancer } \\
\text { histology (\%) }\end{array}$ & $\begin{array}{l}\text { Percentage of } \\
\text { patients with } \\
\text { bone/spinal } \\
\text { metastasis }\end{array}$ & $\begin{array}{l}\text { Signs and } \\
\text { symptoms } \\
\text { of distant } \\
\text { metastasis/indication } \\
\text { for intervention }\end{array}$ & $\begin{array}{l}\text { Intervention for } \\
\text { distant } \\
\text { metastasis } \\
\text { (\% of patients) }\end{array}$ & $\begin{array}{l}\text { Other } \\
\text { metastases (\%) }\end{array}$ & Main outcome(s) \\
\hline $\begin{array}{l}\text { Kloos et al., } \\
2009\end{array}$ & $\begin{array}{l}\text { Total } 56 \text { (44.6\%): } \\
\text { Treatment group } \\
19(42 \%) ; \\
\text { Control group } \\
37(45.9 \%)\end{array}$ & - & $\begin{array}{l}\text { Papillary (73\%), } \\
\text { Follicular (4\%), } \\
\text { Hurthle cell }(16 \%), \\
\text { Anaplastic }(7 \%)\end{array}$ & BM (21\%) & $\begin{array}{l}\text { Evaluate the activity } \\
\text { of sorafenib in } \\
\text { metastatic thyroid } \\
\text { carcinoma }\end{array}$ & $\begin{array}{l}\text { Treatment group; } \\
\text { sorafenib } \\
400 \text { mg PO bd }\end{array}$ & $\begin{array}{l}\text { Lung (96), } \\
\text { lymph (94\%), } \\
\text { other (14\%) }\end{array}$ & $\begin{array}{l}\text { Partial response in } \\
15 \% \text { of patients } \\
\text { ( } 7.5 \text { months } \\
\text { median duration). } \\
\text { Median } \\
\text { progression-free } \\
\text { survival } \\
\text { was } 15 \text { months }\end{array}$ \\
\hline $\begin{array}{l}\text { Cabanillas et al., } \\
2010\end{array}$ & $15(60 \%)$ & $61(38-83)$ & $\begin{array}{l}\text { PWD (53.3\%), } \\
\text { FWD (33.3\%), } \\
\text { Hurthle (13.3\%) }\end{array}$ & BM (26.7\%) & $\begin{array}{l}\text { Evaluate the } \\
\text { activity of sorafenib } \\
\text { and sunitinib in } \\
\text { progressive and } \\
\text { radioactive resistant } \\
\text { DTC }\end{array}$ & $\begin{array}{l}\text { Sorafenib } \\
200-400 \mathrm{mg} \\
\text { PO bd } 86.7 \% \text {, } \\
\text { sunitinib } 50 \mathrm{mg} \\
\text { PO od } 13.3 \%\end{array}$ & $\begin{array}{l}\text { Lymph }(73.3 \%), \\
\text { lung }(66.7 \%) \\
\text { pleura }(13.3 \%)\end{array}$ & $\begin{array}{l}\text { SD } 9(60 \%), \\
\text { PR } 3(20 \%), \\
\text { PD } 3(20 \%) \\
2 \text { yr survival } 67 \%\end{array}$ \\
\hline $\begin{array}{l}\text { Orita et al., } \\
2011\end{array}$ & $\begin{array}{l}\text { Total } 50 \text { (72\%): } \\
\text { Treatment group } \\
22(68.2 \%) ; \\
\text { Control } 28 \text { (75\%) }\end{array}$ & $59(32-77)$ & $\begin{array}{l}\text { PWD (52\%), } \\
\text { FWD (48\%) }\end{array}$ & $\begin{array}{l}\text { SM (40\%), } \\
\text { BM (100\%) }\end{array}$ & $\begin{array}{l}\text { Evaluate the efficacy } \\
\text { of ZA for treatment } \\
\text { of symptomatic BM } \\
\text { in DTC }\end{array}$ & $\begin{array}{l}\text { Treatment group; } \\
\text { ZA } 4 \text { mg } \\
\text { IV/month (100\%), } \\
\text { Surgery (18\%), } \\
\text { ERT (77\%), } \\
\text { RAI (50\%) }\end{array}$ & Others (60\%) & $\begin{array}{l}\text { Treatment } \\
\text { group/control } \\
\text { SRE-free } 3 \text { yr } \\
\text { survival: } \\
86 \% / 50 \% \\
\text { mortality } 54 \%\end{array}$ \\
\hline $\begin{array}{l}\text { Wells et al., } \\
2012\end{array}$ & $\begin{array}{l}\text { Total } 331(42.6 \%): \\
\text { Treatment group } \\
231(42 \%) ; \\
\text { Control group } \\
100(44 \%)\end{array}$ & - & $\begin{array}{l}\text { Advanced MTC } \\
(100 \%)\end{array}$ & $\begin{array}{l}\text { Treatment group } \\
\text { BM (34\%), } \\
\text { control group } \\
\text { BM (40\%) }\end{array}$ & $\begin{array}{l}\text { Evaluate the efficacy } \\
\text { of vandetanib in } \\
\text { advanced or } \\
\text { metastatic MTC }\end{array}$ & $\begin{array}{l}\text { Treatment group; } \\
\text { vandetanib } 300 \text { mg PO }\end{array}$ & $\begin{array}{l}\text { Hepatic }(46.5 \%), \\
\text { lymph nodes }(40.8 \%), \\
\text { respiratory }(38.1 \%), \\
\text { neck }(10.0 \%)\end{array}$ & $\begin{array}{l}\text { Significant } \\
\text { prolongation } \\
\text { of PFS with } \\
\text { vandetanib } \\
\text { compared } \\
\text { with placebo. } \\
\text { PD 37\%, } \\
\text { mortality 15\% }\end{array}$ \\
\hline
\end{tabular}

DTC; differentiated thyroid carcinoma, PWD; papillary well differentiated, FWD; follicular well differentiated, MTC; medullary thyroid carcinoma, PDTC; poorly differentiated thyroid carcinoma, FLD; follicular less differentiated, UTC; undifferentiated thyroid carcinoma, T; thoracic, L; lumbar, C; cervical, BM; bone metastasis, SM; spinal metastasis, SSC; spinal cord compression, RAl; radioactive iodine (131-I), ERT; external

radiotherapy, CT; chemotherapy, SET; selective embolization therapy, TES; total en bloc spondylectomy, ZA; zolendronic acid, NED; no evidence of disease, SD; stable disease, CR; complete remission, PR; partial

response, PD; progressive disease, AWD; alive with disease, DOD; death of disease, SRE; skeletal-related events, PFS; progression-free survival, DSS; disease-specific survival, yr; year. 


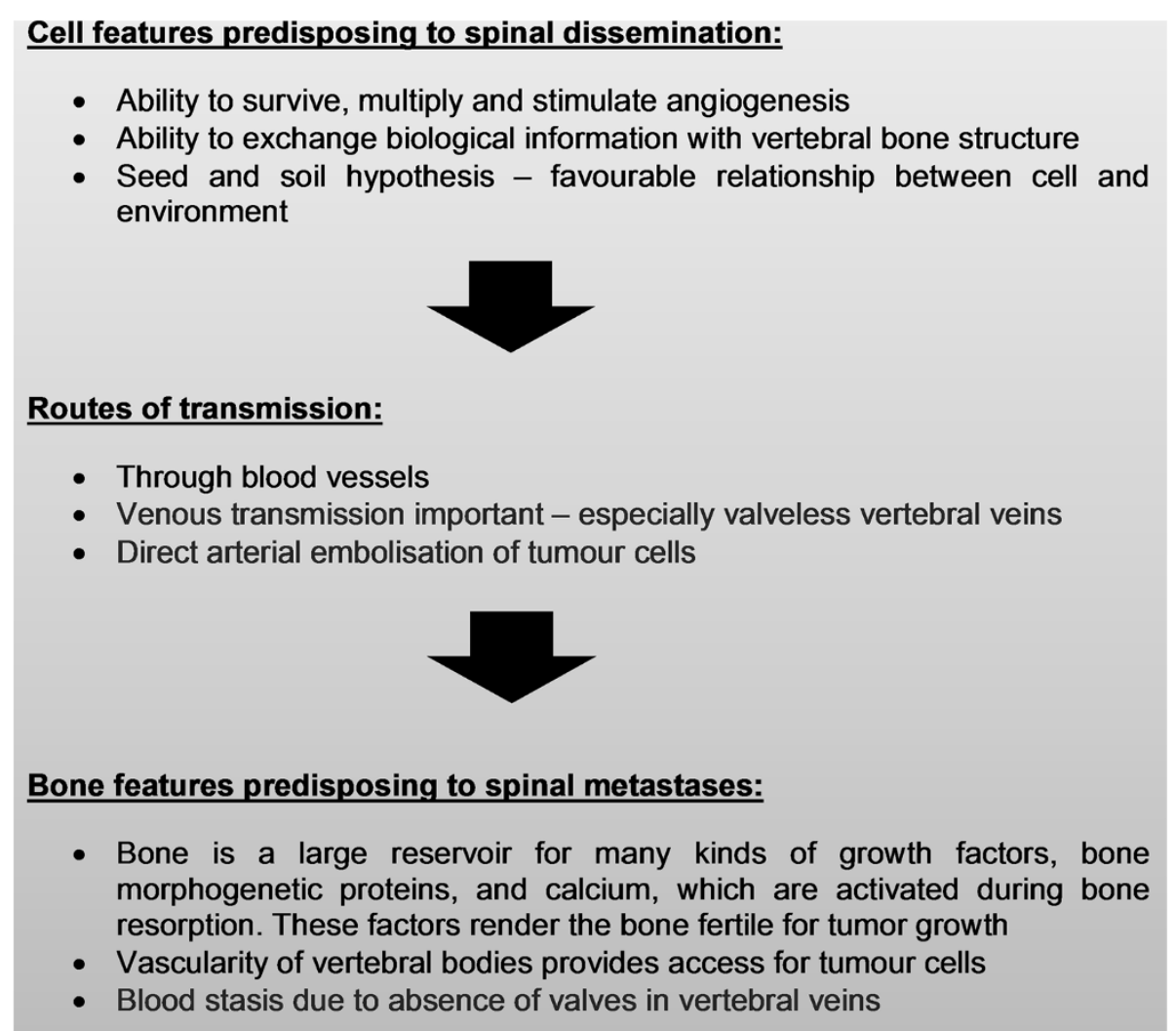

Figure 2 Route of transmission from thyroid to spine.

also mentioned percutaneous vertebroplasty for patients who cannot be operated on but at risk of fractures.

\section{Bisphosphonates}

Bisphosphonates have shown some clinical usefulness in metastatic bone disease in terms of symptom control. Orita et al. [15] illustrated that skeletal related events occurred in significantly lower frequency in patients on zoledronic acid ( $3 / 22$ patients, $14 \%)$ than without (14/28 patients, 50\%; $\mathrm{p}>0.007)$. Pamidronate was also effective at pain amelioration [45], with a $31.35 \%$ reduction using the VAS scoring. Patient tolerability was good, although the side effect of osteonecrosis of the jaw is, unfortunately, relatively high at 9\% [15], though the study size was small. Other studies have intimated that as much as $5 \%$ of the oncological patients receiving bisphosphonate therapy for $\sim 4.4$ years develop bisphosphonate-related osteonecrosis of the jaw (BRONJ) [52]. The authors reported an impressive spinal cord compression reduction, although study size was small $(n=28)$.

\section{Small molecules}

The effect of small molecule inhibitors has revolutionised the treatment of some cancers, and the search of molecular targets in neoplasm continues. Growth signaling pathways are implicated in tumorigenesis, especially those of angiogenesis. Vascular endothelial growth factor receptors (VEGFR) inhibitors appear not to improve progression free survival in metastatic thyroid cancer [37]. However, small study sizes have impeded research. In a study by Kloos et al. [37], side effects prompted 52\% of patients to reduce their dose. Cabanillas et al. [35], treated 15 patients, mostly with non ${ }^{131} \mathrm{I}$ avid disease with different VEGFR inhibitors, notably sorafenib. Interestingly, progression free survival was increased. It was observed that patients whom had their SM irradiated, prior to VEGF therapy, maintained stable bone disease, whereas those who did not have irradiation experienced rapid progression, despite a good response of their lung metastasis to VEGF therapy. This raises the possibility that treating patients with (external bean radiation therapy) EBRT prior to VEGF therapy could improve survival. This may need to be examined in further detail.

\section{Difficulties encountered}

We encountered difficulties with regards to study selection and data extraction, contemporaneous research, study size and consistency of protocol. We cannot claim to have used a complete and exhaustive list of studies. Our search results yielded a large body of research relevant to our 


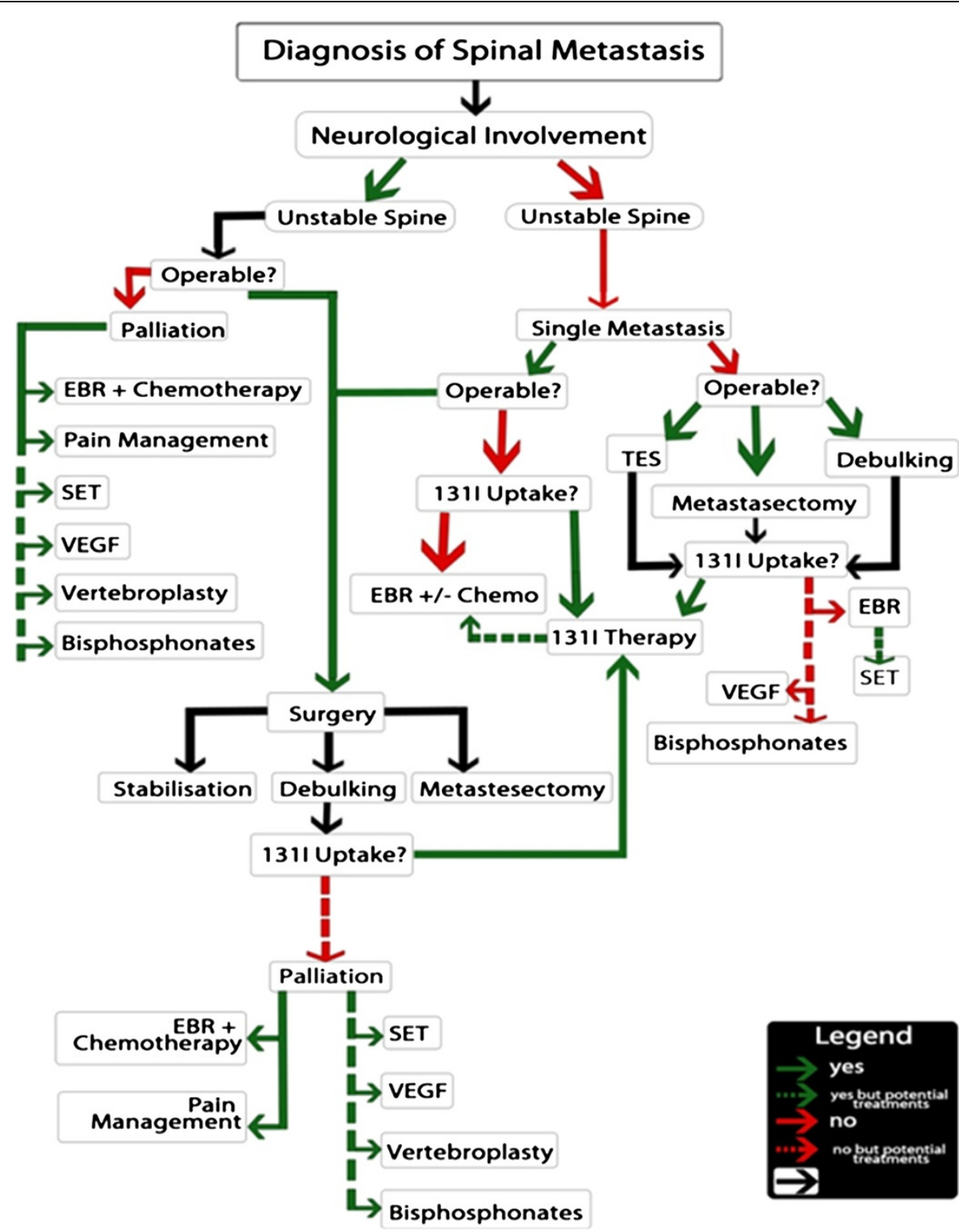

Figure 3 The proposed recommendations of treatment.

title. We cross-examined the references to add to this body. This approach is rigorous but not consummate.

In addition, many of the papers made analysis difficult due to the presentation of their data. Spinal metastasis was rarely isolated as a subgroup. Indeed, many forms of metastases were pooled for the statistics. It was, therefore, impossible to extract specific and valuable information from these studies $[35,37,46]$.

Several retrospective studies did not use contemporary data sets. Studies, recruited by the inclusion criteria, analysed investigation and treatment modalities in a patient pool, which was considerably dated due to some cohorts having been accrued over decades. These studies spanned a long period of time, during which, treatment and investigative modalities had significantly evolved. They, therefore, encompassed a number of out-dated clinical practices and ignored currently well-established modalities [29,30,34,42].

Much of the literature on spinal metastasis in thyroid cancer is based on small sample sized studies. This makes it difficult to measure the effectiveness of some treatment modalities and draw conclusions. Additionally, this makes it difficult to use the data in multivariate analysis due to the high standard errors, which would occur with small sample size $[14,16,36,40,44,45]$.

Finally, most countries have specific guidelines and recommendations for the management of certain cancers, including the UK [19]. It was difficult to make 
recommendations when papers preconised the use of different 'successful' methods in similar situations. This applied to certain treatments, such as thyroidectomy, or to dose, such of ${ }^{131}$ I used $[14,16,19,40]$.

\section{Recommendations}

Individual prognosis depends upon age at diagnosis of spinal metastasis, tumour burden and number of spinal metastases $[29,30,34,42,47]$. Early detection is a prognostic factor in spinal metastasis of differentiated thyroid carcinoma (DTC). This early detection could help prevent dissemination to the spine. The proposed process of which is outlined in figure 2. Post-surgical ${ }^{131} \mathrm{I}$ ablation, in addition to adjuvant therapy, has been shown to aid in the early detection of bone metastasis. At this time, thyroglobulin levels are low, and radiological studies are often negative [14]. Treatment of metastasis should be done in a multi-disciplinary fashion and take into account tumour response, palliation and neurological function. Recommendations of treatment are illustrated in Figure 3.

Radioiodine absorption is a prognostic factor in metastatic disease. Multivariate analysis expounds the prognostic benefit of complete metastectomy and ${ }^{131} \mathrm{I}$ therapy, when applicable. Survival rates in DTC patients are improved by these interventions $[13,34,38,47]$. This is, especially so in those below the age of 45 , in whom bone is the only distant metastasis [34]. We espouse that therapy should be aggressive, particularly in the young [34].

Patients below the age of 45 years of age with small DTC distant metastasis should be treated with radioiodine until there is an uptake decline or a cumulative dose of $600 \mathrm{mCi}(22 \mathrm{GBq}$ ) reached [30]. Subsequent courses of radioiodine treatment, at least one year after initial radioiodine treatment may also be a recommended.

Radioiodine therapy comes with its limitations, however. Treatment exceeding $600 \mathrm{mCi}$ may be deleterious to the health. There is a significant increase in salivary gland dysfunction, cancer and leukaemia in patients. In patients with continued uptake, a decision to continue treatment beyond that dose should be considered on a case-by-case basis, due the risks associated [30].

In patients with non ${ }^{131} \mathrm{I}$ avid disease, or who are ${ }^{131} \mathrm{I}$ non-responders, other treatment modalities ought to be evaluated. Such treatments include combination therapy, SET or small molecules, along with radiotherapy. These may assuage symptomatic disease. VEGFR is particularly helpful in ${ }^{131}$ I non-avid disease. SET and bisphosphonates are especially useful modalities in reducing bone pain $[15,16,44,45]$.

Thyroid cancer pathogenesis and promulgation to the bone has yet to be fully elucidated despite some novel research by Torre et al. [53] into the role of angiogenic and lymphangiogenic phenotypes, spread to the vertebral column is poorly understood. Dissection of this journey opens the possibility of intervening to improve patient survival and reduced morbidity.

\section{Conclusions}

There is a need for more histology-specific research in the field of thyroid carcinoma metastasis. While study sizes are difficult to control, we believe that there is room for improvement in study design and in the quantity of research on specific treatment modalities.

To sum up, for a young patient aggressive surgery is the best first line management. Radioiodine and surgery is our best combined management. Combination therapy, SET or small molecular inhibitor with radiation is recommended.

Furthermore, VEGFR is particularly helpful in ${ }^{131} \mathrm{I}$ non-avid disease. SET and bisphosphonates are useful modalities in palliation. However, more research is required into the effect of combination therapies.

Future prospects look towards intersecting the molecular pathways of tumourigenesis and dissemination. Indeed, spread to the spine is haematogenous, which opens an avenue to pursue. At present, surgery is our most logical solution, but it certainly is not curative, but, rather, symptom control. Cure is the aim.

\section{Competing interests}

The authors declare that they have no competing interests.

\section{Authors' contribution}

All authors contributed to conception and design, carried out the literature research, manuscript preparation and manuscript review. All authors read and approved the final manuscript

\section{Author details}

${ }^{1}$ Department of Medicine, University College London Medical School, London, UK. 'Barts and The London School of Medicine and Dentistry, University of London, Queen Mary, London, UK. ${ }^{3}$ Department of Surgery, Al-Yarmouk University College, Baghdad, Iraq. ${ }^{4}$ Department of Surgery, UCL Medical School, London, UK. ${ }^{5}$ Leeds Institute of Molecular Medicine, University of Leeds, London, UK. ${ }^{6}$ Academic Department of Trauma and Orthopaedic Surgery, Leeds Teaching Hospitals NHS Trust, Leeds, UK.

Received: 6 June 2012 Accepted: 7 June 2012

Published: 25 June 2012

\section{References}

1. Batson OV: The function of the vertebral veins and their role in the spread of metastases. Ann Surg 1940, 112:138-149.

2. Roodman GD: Mechanisms of disease: Mechanisms of bone metastasis. N Engl J Med 2004, 350:1655-1664.

3. Muresan MM, et al: Bone metastases from differentiated thyroid carcinoma. Endocr Relat Cancer 2008, 15:37-49.

4. Sherman SI: Thyroid carcinoma. Lancet 2003, 361:501-511.

5. Younes MN, et al: Antivascular therapy of human follicular thyroid cancer experimental bone metastasis by blockade of epidermal growth factor receptor and vascular growth factor receptor phosphorylation. Cancer Res 2005, 65:4716-4727.

6. Aschebrook-Kilfoy B, Ward MH, Sabra MM, Devesa SS: Thyroid cancer incidence patterns in the United States by histologic type, 1992-2006. Thyroid 2011, 21:125-134. 
7. Kilfoy BA, et al: International patterns and trends in thyroid cancer incidence, 1973-2002. Cancer Causes Control 2009, 20:525-531.

8. Prasad D, Schiff D: Malignant spinal-cord compression. Lancet Oncol 2005, 6:15-24.

9. Thomas WH, Coppola ED: Distant metastases from mixed tumors of the salivary glands. Am J Surg 1965, 109:724-730.

10. Baudin E, Schlumberger M: New therapeutic approaches for metastatic thyroid carcinoma. Lancet Oncol 2007, 8:148-156.

11. Wilson PC, Millar BM, Brierley JD: The management of advanced thyroid cancer. Clin Oncol (R Coll Radiol) 2004, 16:561-568.

12. Sciubba DM, et al: Solitary spinal metastasis of Hürthle cell thyroid carcinoma. J Clin Neurosci 2010, 17:797-801.

13. Demura $\mathrm{S}$, et al: Total en bloc spondylectomy for spinal metastases in thyroid carcinoma. J Neurosurg Spine 2011, 14:172-176.

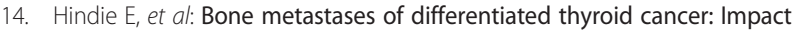
of early 1-131-based detection on outcome. Endocr Relat Cancer 2007, 14:799-807.

15. Orita Y, Sugitani I, Toda K, Manabe J, Fujimoto Y: Zoledronic acid in the treatment of bone metastases from differentiated thyroid carcinoma. Thyroid 2011, 21:31-35.

16. Eustatia-Rutten CF, et al: Outcome of palliative embolization of bone metastases in differentiated thyroid carcinoma. J Clin Endocrinol Metab 2003, 88:3184-3189.

17. Cooper DS, et al: Management guidelines for patients with thyroid nodules and differentiated thyroid cancer. Thyroid 2006, 16:109-142.

18. Sherman SI: The management of metastatic differentiated thyroid carcinoma. Rev Endocr Metab Disord 2000, 1:165-171.

19. Physicians, R. C. o: In Edited by British Thyroid Association. London: Royal College of Physicians; 2007.

20. Williams SD, Birch R, Einhorn LH: Phase-ii evaluation of doxorubicin plus cisplatin in advanced thyroid-cancer - a southeastern-cancer-study -group trial. Cancer Treat Rep 1986, 70:405-407.

21. Mazzaferri EL, Jhiang SM: Long-term impact of initial surgical and medical therapy on papillary and follicular thyroid-cancer. Am J Med 1994, 97:418-428.

22. Vassilopoulou-Sellin R, Goepfert H, Raney B, Schultz PN: Differentiated thyroid cancer in children and adolescents: Clinical outcome and mortality after long-term follow-up. Head and Neck-Journal for the Sciences and Specialties of the Head and Neck 1998, 20:549-555.

23. Kushchayev S, Kushchayeva Y, Theodore N, Preul MC, Clark OH: Percutaneous vertebroplasty for thyroid cancer metastases to the spine. Thyroid 2010, 20:555-560.

24. Tickoo SK, et al: Bone metastases from thyroid carcinoma: a histopathologic study with clinical correlates. Arch Pathol Lab Med 2000, 124:1440-1447.

25. Harel $R$, Angelov L: Spine metastases: current treatments and future directions. Eur J Cancer 2010, 46:2696-2707.

26. In N, E. Vol. Edited by Newey M.: Surg Oncol; 2012.

27. Molina CA, Gokaslan ZL, Sciubba DM: Diagnosis and management of metastatic cervical spine tumors. Orthop Clin N Am 2012, 43:75.

28. Perrin RG, Laxton AW: Metastatic spine disease: epidemiology, pathophysiology, and evaluation of patients. Neurosurg Clin N Am 2004, 15:365-+.

29. Pittas $A G$, et al: Bone metastases from thyroid carcinoma: clinical characteristics and prognostic variables in one hundred forty-six patients. Thyroid 2000, 10:261-268.

30. Durante $C$, et al: Long-term outcome of 444 patients with distant metastases from papillary and follicular thyroid carcinoma: benefits and limits of radioiodine therapy. J Clin Endocrinol Metab 2006, 91:2892-2899.

31. Wexler JA: Approach to the Thyroid Cancer Patient with Bone Metastases. J Clin Endocrinol Metab 2011, 96:2296-2307.

32. Sciubba DM, et al: Diagnosis and management of metastatic spine disease A review. J Neurosurg Spine 2010, 13:94-108.

33. Harrington KD: Metastatic disease of the spine. Journal of Bone and Joint Surgery-American 1986, 68A:1110-1115.

34. Bernier $\mathrm{MO}$, et al: Survival and therapeutic modalities in patients with bone metastases of differentiated thyroid carcinomas. J Clin Endocrinol Metab 2001, 86:1568-1573.

35. Cabanillas ME, et al: Treatment with tyrosine kinase inhibitors for patients with differentiated thyroid cancer: the M. D. Anderson experience. J Clin Endocrinol Metab 2010, 95:2588-2595.
36. De Vries MM, et al: Embolization therapy of bone metastases from epithelial thyroid carcinoma: effect on symptoms and serum thyroglobulin. Thyroid 2008, 18:1277-1284.

37. Kloos RT, et al: Phase II trial of sorafenib in metastatic thyroid cancer. J Clin Oncol 2009, 27:1675-1684

38. Orita $Y$, et al: Prognostic factors and the therapeutic strategy for patients with bone metastasis from differentiated thyroid carcinoma. Surgery 2010, 147:424-431.

39. Petrich $T$, et al: Outcome after radioiodine therapy in 107 patients with differentiated thyroid carcinoma and initial bone metastases: side-effects and influence of age. Eur J Nucl Med 2001, 28:203-208.

40. Quan GM, Pointillart V, Palussière J, Bonichon F: Multidisciplinary treatment and survival of patients with vertebral metastases from thyroid carcinoma. Thyroid 2012, 22:125-130.

41. Qiu ZL, Song HJ, Xu YH, Luo QY: Efficacy and survival analysis of 131 I therapy for bone metastases from differentiated thyroid cancer. J Clin Endocrinol Metab 2011, 96:3078-3086.

42. Schlumberger $M$, et al: Radioactive iodine treatment and external radiotherapy for lung and bone metastases from thyroid carcinoma. J NuCl Med 1996, 37:598-605.

43. Stojadinovic A, et al: The role of operations for distantly metastatic welldifferentiated thyroid carcinoma. Surgery 2002, 131:636-643.

44. Van Tol KM, et al: Embolization in combination with radioiodine therapy for bone metastases from differentiated thyroid carcinoma. Clin Endocrinol (Oxf) 2000, 52:653-659.

45. Vitale $G$, et al: Pamidronate improves the quality of life and induces clinical remission of bone metastases in patients with thyroid cancer. Br J Cancer 2001, 84:1586-1590.

46. Wells SA, et al: Vandetanib in patients with locally advanced or metastatic medullary thyroid cancer: a randomized, double-blind phase III trial. J Clin Oncol 2012, 30:134-141.

47. Zettinig $\mathrm{G}$, et al: Long-term follow-up of patients with bone metastases from differentiated thyroid carcinoma - surgery or conventional therapy? Clin Endocrinol (Oxf) 2002, 56:377-382.

48. Tokuhashi Y, Ajiro Y, Oshima M: Algorithms and Planning in Metastatic Spine Tumors. Orthop Clin N Am 2009, 40:37-+.

49. Hosono N, Ueda T, Tamura D, Aoki Y, Yoshikawa H: Prognostic relevance of clinical symptoms in patients with spinal metastases. Clin Orthop Relat Res 2005, 196-201.

50. Mercadante S, Fulfaro F: Management of painful bone metastases. Curr Opin Oncol 2007, 19:308-314.

51. Sayama CM, Schmidt MH, Bisson EF: Cervical spine metastases: techniques for anterior reconstruction and stabilization. Neurosurg Rev 2012

52. Clarke BM, et al: Bisphosphonates and jaw osteonecrosis: the UAMS experience. Otolaryngol Head Neck Surg 2007, 136:396-400.

53. de la Torre NG, Buley I, Wass JA, Turner HE: Angiogenesis and lymphangiogenesis in thyroid proliferative lesions: relationship to type and tumour behaviour. Endocr Relat Cancer 2006, 13:931-944.

54. Costachescu B, Popescu CE: Modern management in vertebral metastasis. Romanian Neurosurgery. 2010, 27(4):432-437.

doi:10.1186/1758-3284-4-39

Cite this article as: Ramadan et al.: Spinal metastasis in thyroid cancer. Head \& Neck Oncology 2012 4:39.

\section{Submit your next manuscript to BioMed Central and take full advantage of:}

- Convenient online submission

- Thorough peer review

- No space constraints or color figure charges

- Immediate publication on acceptance

- Inclusion in PubMed, CAS, Scopus and Google Scholar

- Research which is freely available for redistribution 